\title{
Non-fragile $H_{\infty}$ Fuzzy Filtering with Randomly Occurring Gain Variations and Channel Fadings
}

\author{
Sunjie Zhang, Zidong Wang, Derui Ding, Hongli Dong, Fuad E. Alsaadi and Tasawar Hayat
}

\begin{abstract}
This paper is concerned with the non-fragile $H_{\infty}$ filtering problem for a class of discrete-time T-S fuzzy systems with both randomly occurring gain variations (ROGVs) and channel fadings. The phenomenon of the ROGVs is introduced into the system model so as to account for the parameter fluctuations occurring during the filter implementation. Two sequences of random variables obeying the Bernoulli distribution are employed to describe the phenomenon of the ROGVS bounded by prescribed norms. Also, the Rice fading model is utilized to describe the phenomena of channel fadings where the occurrence probabilities of the random channel coefficients are allowed to time-varying. Through stochastic analysis and Lyapunov functional approach, sufficient conditions are established under which the filtering error dynamics is exponentially meansquare stable with a prespecified $H_{\infty}$ performance. The set of the desired non-fragile $H_{\infty}$ filters is characterized by solving a convex optimization problem via the semi-definite programming method. An illustrative example is given to show the usefulness and effectiveness of the proposed design method in this paper.
\end{abstract}

Index Terms-Discrete-time systems, stochastic systems, fuzzy systems, non-fragile $H_{\infty}$ filter, randomly occurring gain variations, channel fadings.

\section{INTRODUCTION}

As is well known, almost all real-world dynamical systems involve inherently nonlinear phenomena and the nonlinear control problem has long been a focus of research attracting persistent attention from a wide range of communities. Over the past few decades, fuzzy logic theory has proven to be effective in dealing with a variety of complex nonlinear systems, and a wealth of research results has been reported, see e.g. [21], [25], [36], [39] and the references therein. Among different kinds of models for fuzzy systems, the socalled Takagi-Sugeno (T-S) fuzzy model has been one of the most popular models due mainly to its convenient and simple dynamic structure. More specifically, the T-S fuzzy model is capable of utilizing fuzzy rules to approximate a global

This work was supported in part by the National Natural Science Foundation of China under Grants 61134009, 61329301, 61203139, 61374127 and 61422301, the Hujiang Foundation of China under Grant C14002, the Royal Society of the U.K., and the Alexander von Humboldt Foundation of Germany.

S. Zhang and D. Ding are with the Shanghai Key Lab of Modern Optical System, Department of Control Science and Engineering, University of Shanghai for Science and Technology, Shanghai 200093, China.

Z. Wang is with the Department of Computer Science, Brunel University London, Uxbridge, Middlesex, UB8 3PH, United Kingdom. He is also with the Faculty of Engineering, King Abdulaziz University, Jeddah 21589, Saudi Arabia. (Email: Zidong.Wangebrunel.ac.uk)

H. Dong is with the College of Electrical and Information Engineering, Northeast Petroleum University, Daqing 163318, China.

F. E. Alsaadi and T. Hayat are with the Faculty of Engineering, King Abdulaziz University, Jeddah 21589, Saudi Arabia. T. Hayat is also with the Department of Mathematics, Quaid-i-Azam University, Islamabad, Pakistan. nonlinear system by means of a set of local linear models that are smoothly connected by fuzzy membership functions [41], [42]. This model could also exactly represent a family of nonlinear systems over a compact subspace by means of the sector nonlinearity approach [43]. As a result, the conventional linear system theory can be applied to analysis and synthesis of the class of nonlinear control systems. Up to now, the T-S fuzzy control and filtering have been extensively studied and many significant results have been available in the literature, see e.g. [4], [9], [11], [13], [18].

Filtering problems have been a main stream of research for nearly 60 years because of their practical insights and applications in a variety of areas such as military, astronautics and signal processing [37], [48]. Filtering problems have drawn considerable research attention from both scientists and engineers and, so far, many different kinds of methodologies have been developed to handle the filtering issues with various complex systems. When the statistical information on the external noise signals is unknown a priori, the classical Kalman filtering approach cannot be directly applied since it requires exact knowledge about the noise statistics. As an effective alternative, the $H_{\infty}$ filtering algorithm has been introduced to deal with the energy-bounded external noise signals, where the main objective is to constrain the $H_{\infty}$ norm (from the process noises to the filter errors) to an acceptable attenuation level [27]. Apart from the disturbance attenuation behavior, the $H_{\infty}$ filter has been shown to be robust against unmodeled dynamics [51]. In the past few years, the $H_{\infty}$ filtering techniques based on the T-S fuzzy models have gained a great deal of research interest and much work has been done on this issue, see e.g., [10], [26], [32], [33], [45], [50].

Note that all the aforementioned results concerning filtering problems are based on an implicit assumption that the filters can be implemented precisely. However, fluctuations or drifts may occur in the implementation of the designed filter even though the filter is well designed. This is due to unexpected errors during the filter implementation, e.g., analog-digital and digital-analog conversion, finite word length, finite resolution measuring instruments, programming errors, roundoff errors in numerical computations, the need to provide practicing engineers with safe-tuning margins, and so on. Such fluctuations, though small, should not be simply neglected as they might have a significant impact on the system behaviors [24]. As such, the filter should be designed to be insensitive against certain errors/variations with respect to its gain, i.e., the designed filter is expected to be insensitive or non-fragile. Currently, both the deterministic and persistent uncertainties have been utilized to quantify the controller/filter gain vari- 
ations, see [5], [6], [12], [28], [29], [49] for more details. In particular, in a networked situation where the filter is realized through a communication network, the occurrence of the filter gain variations may be subject to random changes due to environmental circumstances. In this case, the gain variations may be present in a probabilistic way with certain types and intensity. As such, the phenomenon of randomly occurring gain variations (ROGVs) should be taken into account when designing filters. Note that, very recently, a non-fragile controller with ROGVs has been studied in [17] to address the synchronization problem of neural networks, but the corresponding non-fragile fuzzy filter problem with ROGVs has not received adequate research attention yet.

Because of the ever-increasing popularity of communication networks, the signals are often transmitted through networks which could result in communication delays, packet dropouts and signal quantizations. Furthermore, in wireless communication networks, the electromagnetic waves might not directly reach the filter due probably to the obstacles blocking the line of sight path [31]. In this case, a signal could travel from transmitter to receiver over a multiple-reflection path which gives rise to the channel fading phenomenon. In reality, it is known that wireless channels are sensitive to fading effects [15], and this constitutes one of the most dominant features in wireless communication links. The fading phenomenon is often modeled as a time-varying stochastic mathematical model so as to represent the transmitted signal's change in both the amplitude and phase. Concerning the system measurements transmitted via fading channels, much effort has been devoted to the stability analysis, simulation study and LQG control problems, see [15], [16], [34], [47] and the references therein. For instance, the remote mean-square stabilization problem has been thoroughly investigated in [15] for various fading channels including the analog erasure channel as well as the $L$ th-order Rice fading channel. Nevertheless, when the channel fading issue is coupled with the ROGV phenomenon, the nonfragile filtering problem is still an open one that deserves intensive investigation, especially for discrete-time T-S fuzzy systems with $H_{\infty}$ performance constraints.

Motivated by the aforementioned discussion, a seemingly natural problem arises, that is, how to design the filters for certain discrete-time nonlinear systems subject to ROGVs in the presence of measurement transmission over a fading channel? In [5], the non-fragile filter design problem has been studied for a class of continuous-time fuzzy systems, and the problem of non-fragile $H_{\infty}$ filtering has been dealt with in [12] for a class of discrete-time T-S fuzzy systems. Note that the non-fragile filtering have been considered in these references. So far, to the best of the authors' knowledge, the non-fragile $H_{\infty}$ filtering problem for T-S fuzzy system with ROGVs and channel fadings has not been investigated yet, and the main purpose of this paper is to shorten such a gap by making the initiatives.

The main contributions of this paper can be highlighted as follows. 1) A fairly comprehensive system model is proposed to describe the ROGVs conforming to Bernoulli distributions. 2) A modified Rice fading model, whose channel coefficients obey time-varying probabilities with known bounds, is considered in the design of the filter in order to better reflecting the reality of measurement transmissions especially via a wireless sensor network. 3) A unified framework is established to deal with a class of complex systems accounting for both the ROGVs and the channel fadings via stochastic analysis and matrix manipulations. The rest of this paper is organized as follows. In Section II, the model for the problem under consideration is presented and some assumptions are made on the ROGVs and the channel coefficients. Sufficient conditions are obtained in Section III on the exponential stability and $H_{\infty}$ performance for the filtering error dynamics. An illustrative example is provided in Section IV to demonstrate the effectiveness of the main results. Section V concludes the paper.

Notation. The notation used in the paper is fairly standard. The superscript " $T$ " stands for matrix transposition, $\mathbb{R}^{n}$ denotes the $n$-dimensional Euclidean space, and $\mathbb{R}^{m \times n}$ is the set of all real matrices of dimension $m \times n$. $I$ and 0 represent the identity matrix and zero matrix, respectively. The notation $P>0$ means that $P$ is real, symmetric and positive definite. $l_{2}\left([0,+\infty), \mathbb{R}^{n}\right)$ is the space of square summable sequences. $\operatorname{tr}(M)$ refers to the trace of the matrix $M$. The notation $\|A\|$ refers to the Frobenius norm of a matrix $A$ defined by $\|A\|=\sqrt{\operatorname{tr}\left(A^{T} A\right)}$. In symmetric block matrices or complex matrix expressions, we use an asterisk $(*)$ to represent a term that is induced by symmetry, and $\operatorname{diag}\{\ldots\}$ stands for a block-diagonal matrix. The symbol $\otimes$ denotes the Kronecker product. Matrices, if their dimensions are not explicitly stated, are assumed to be compatible for algebraic operations.

\section{Problem Formulation and Preliminaries}

In this paper, we consider the following nonlinear discretetime system represented by T-S fuzzy dynamic model, in which the rule is described as follows:

Plant Rule $i$ : IF $\theta_{1}(k)$ is $\eta_{i 1}$, and $\theta_{2}(k)$ is $\eta_{i 2}$, and ..., and $\theta_{r}(k)$ is $\eta_{i r}$, THEN

$$
\left\{\begin{aligned}
x(k+1)= & A_{i} x(k)+G_{i} x(k-\tau(k))+B_{i} w(k) \\
y(k)= & C_{i} x(k)+D_{i} w(k) \\
z(k)= & E_{i} x(k) \\
x(s)= & \phi(s), \quad i \in \mathcal{R} \triangleq\{1,2, \cdots, r\}, \\
& \forall s \in \mathcal{S} \triangleq\left\{-\tau_{M},-\tau_{M}+1, \cdots, 0\right\}
\end{aligned}\right.
$$

where $r$ is the number of IF-THEN rules, $\eta_{i j}(i, j=$ $1,2, \cdots, r)$ is the fuzzy set, the premise variable vector is represented by $\theta(k)=\left[\theta_{1}(k), \theta_{2}(k), \cdots, \theta_{r}(k)\right] . x(k) \in \mathbb{R}^{p}$ is the state vector, $y(k) \in \mathbb{R}^{s}$ is the measurement output, $z(k) \in \mathbb{R}^{q}$ is the signal to be estimated, $w(k) \in l_{2}[0,+\infty)$ is the disturbance input, and $\phi(s)$ is the initial state. $\tau(k)$ is known time-varying delays satisfying $0 \leq \tau_{m} \leq \tau(k) \leq \tau_{M}$ where $\tau_{m}$ and $\tau_{M}$ are known positive integers representing the minimum and maximum delays, respectively. The matrices $A_{i}, B_{i}, C_{i}, D_{i}, E_{i}$ and $G_{i}$ are known constant matrices with appropriate dimensions.

Let us now discuss the phenomenon of channel fading for which the channel coefficients have time-varying probability characteristics. In this paper, due to the existence of channel fadings between the physical plant and the filter, the measurements of the plant are no longer equivalent to the inputs of 
the filter. According to Lth-order Rice fading model in [15], the signal received by the filter can be modeled as follows:

$$
y_{f}(k)=\lambda_{0}(k) y(k)+\sum_{l=1}^{L} \lambda_{l}(k) y(k-l),
$$

where $\lambda_{l}(k)(l=0,1, \cdots, L)$ are the channel coefficients, which are I.I.D (independent and identically distributed) Gaussian random variables with $\mathbb{E}\left\{\lambda_{l}(k)\right\}=p_{l}(k)$ and $\mathbb{E}\left\{\lambda_{l}^{2}(k)\right\}=q_{l}(k) . p_{l}(k)$ and $q_{l}(k)$ are two time-varying positive scalar sequences that takes values on the interval $\left[\underline{p}_{l}, \bar{p}_{l}\right]$ and $\left[\underline{q}_{l}, \bar{q}_{l}\right]$ with constants $\underline{p}_{l}, \bar{p}_{l}, \underline{q}_{l}$ and $\bar{q}_{l}$.

It is easy to see that the model (2) could cater for the phenomena of both channel fadings and time-delays at the same time, where $L$ is the order of the Rice fading model. Furthermore, to make the description meaningful, we can set $\ell(k)=\min \{L, k\}$ and then the receiver model (2) can be rewritten as

$$
y_{f}(k)=\lambda_{0}(k) y(k)+\sum_{l=1}^{\ell(k)} \lambda_{l}(k) y(k-l) .
$$

Remark 1: The Lth-order Rice fading model, which is applicable to point-to-point communication systems, is a stochastic one widely used in the area of signal processing. The Rice fading model is capable of accounting for timedelays, packet dropouts and channel fadings. Compared with original Rice fading model in [15], the channel coefficients $\lambda_{l}(k)(l=0,1, \cdots, L)$ are allowed to have time-varying mathematical expectations $p_{l}(k)$ and variances $q_{l}(k)$ in this paper, thereby better reflecting the reality of measurement transmission. As can be seen later, such a signal propagation process leads to substantial difficulties in the subsequent analysis and design.

Next, for the physical plant represented by (1), we take the ROGV phenomenon into account and propose the filter as follows.

Filter Rule $i$ : IF $\theta_{1}(k)$ is $\eta_{i 1}$, and $\theta_{2}(k)$ is $\eta_{i 2}$, and ..., and $\theta_{r}(k)$ is $\eta_{i r}$, THEN

$$
\left\{\begin{aligned}
x_{f}(k+1)= & \left(A_{f i}+\alpha(k) \Delta A_{f i}(k)\right) x_{f}(k) \\
& +\left(B_{f i}+\beta(k) \Delta B_{f i}(k)\right) y_{f}(k) \\
z_{f}(k)= & E_{i} x_{f}(k), \quad i \in \mathcal{R}
\end{aligned}\right.
$$

where $x_{f}(k) \in \mathbb{R}^{n}$ is the state vector of the filter system, $z_{f}(k) \in \mathbb{R}^{q}$ is an estimation of $z(k) . \alpha(k)$ and $\beta(k)$ are mutually independent Bernoulli-distributed white sequences. A natural assumption on $\alpha(k)$ and $\beta(k)$ can be made as follows:

$$
\begin{aligned}
& \operatorname{Prob}\{\alpha(k)=1\}=\mathbb{E}\{\alpha(k)\}=\bar{\alpha}, \\
& \operatorname{Prob}\{\alpha(k)=0\}=1-\bar{\alpha}, \\
& \operatorname{Prob}\{\beta(k)=1\}=\mathbb{E}\{\beta(k)\}=\bar{\beta}, \\
& \operatorname{Prob}\{\beta(k)=0\}=1-\bar{\beta} .
\end{aligned}
$$

Moreover, for each $k$, both $\alpha(k)$ and $\beta(k)$ are independent from $\lambda_{l}(k)(l=0,1, \cdots, L) . A_{f i}$ and $B_{f i}$ are appropriately dimensioned filter matrices to be determined. The uncertain perturbation matrices $\Delta A_{f i}(k)$ and $\Delta B_{f i}(k)$ are defined as follows:

$$
\begin{aligned}
& \Delta A_{f i}(k)=M_{a i} \Delta_{1}(k) N_{a}, \\
& \Delta B_{f i}(k)=M_{b i} \Delta_{2}(k) N_{b}, \quad i \in \mathcal{R},
\end{aligned}
$$

where $M_{a i}, M_{b i}, N_{a}$ and $N_{b}$ are known constant matrices with appropriate dimensions, and $\Delta_{1}(k)$ and $\Delta_{2}(k)$ are unknown matrices function satisfying

$$
\Delta_{r}^{T}(k) \Delta_{r}(k) \leq I, r=1,2 .
$$

Remark 2: In nowadays networked world, the filter parameters may be subject to random changes during the implementation in a networked environment. Such random changes are due to a variety of reasons such as network-induced random failures and repairs of components, sudden environmental disturbances, etc. Very recently, in [22], the concept of randomly occurring uncertainties (ROUs) has been introduced to model the randomly occurring parameter uncertainties for sliding mode control. In comparison with [5] and [12], two random variables $\alpha(k)$ and $\beta(k)$ are introduced to model the ROGVs for the non-fragile filters. Such a description is suitable for catering for the parameter variations of the filter in a random fashion particularly in the signal transmissions through a communication network.

Denote

$$
\vartheta_{i}(\theta(k))=\prod_{j=1}^{r} \eta_{i j}\left(\theta_{j}(k)\right), h_{i}(\theta(k))=\frac{\vartheta_{i}(\theta(k))}{\sum_{j=1}^{r} \vartheta_{j}(\theta(k))},
$$

where $\eta_{i j}\left(\theta_{j}(k)\right)$ is the grade of membership of $\theta_{j}(k)$ in $\eta_{i j}$. It is always assumed that $\vartheta_{i}(\theta(k)) \geq 0(i=1,2, \cdots, r)$ and $\sum_{j=1}^{r} \vartheta_{j}(\theta(k))>0$. Obviously, one has that

$$
h_{i}(\theta(k)) \geq 0, \sum_{j=1}^{r} h_{j}(\theta(k))=1, \forall i \in \mathcal{R}, \forall k \in \mathbb{Z} .
$$

In what follows, we define $h_{i} \triangleq h_{i}(\theta(k))$ for brevity. Then, the defuzzified output of the T-S fuzzy system (1) can be represented as follows:

$$
\left\{\begin{aligned}
x(k+1) & =\sum_{i=1}^{r} h_{i}\left(A_{i} x(k)+G_{i} x(k-\tau(k))+B_{i} w(k)\right) \\
y(k) & =\sum_{i=1}^{r} h_{i}\left(C_{i} x(k)+D_{i} w(k)\right) \\
z(k) & =\sum_{i=1}^{r} h_{i} E_{i} x(k)
\end{aligned}\right.
$$

Moreover, the filter system can also be described by

$$
\left\{\begin{aligned}
x_{f}(k+1)= & \sum_{i=1}^{r} h_{i}\left(\left(A_{f i}+\alpha(k) \Delta A_{f i}(k)\right) x_{f}(k)\right. \\
& \left.+\left(B_{f i}+\beta(k) \Delta B_{f i}(k)\right) y_{f}(k)\right) \\
z_{f}(k)= & \sum_{i=1}^{r} h_{i} E_{i} x_{f}(k)
\end{aligned}\right.
$$


For notational simplicity, in the rest of this paper, we denote

$$
\begin{gathered}
\sum_{a_{1}, a_{2}, \cdots, a_{s}=1}^{r} h_{a_{1}} h_{a_{2}} \cdots h_{a_{s}} \\
\triangleq \sum_{a_{1}=1}^{r} h_{a_{1}} \sum_{a_{2}=1}^{r} h_{a_{2}} \ldots \sum_{a_{s}=1}^{r} h_{a_{s}}, \forall s \geq 1 .
\end{gathered}
$$

The dynamics of the filtering error looks quite complicated because the $L$ th-order Rice fading model leads to some delayed membership functions appearing in the error dynamics. Without any loss of the generality, from now on, we assume that $C_{i}=C$ and $D_{i}=D(i=1,2, \cdots, r)$ in (1). Combining (3), (9) and (10), the filtering error dynamics can be represented as

$$
\left\{\begin{aligned}
\xi(k+1)= & \sum_{i, j=1}^{r} h_{i} h_{j}\left[\left(\bar{A}_{i j}+\bar{\alpha} \Delta \tilde{A}_{j}(k)+\tilde{\alpha}(k) \Delta \tilde{A}_{j}(k)\right.\right. \\
& +\lambda_{0}(k) \tilde{A}_{j}+\bar{\beta} \lambda_{0}(k) \Delta \tilde{A}_{j}(k)+\tilde{\beta}(k) \lambda_{0}(k) \\
& \left.\times \Delta \tilde{A}_{j}(k)\right) \xi(k)+\tilde{G}_{i} \xi(k-\tau(k))+\left(\bar{B}_{i}\right. \\
& +\lambda_{0}(k) \tilde{B}_{j}+\bar{\beta} \lambda_{0}(k) \Delta \tilde{B}_{j}(k)+\tilde{\beta}(k) \lambda_{0}(k) \\
& \left.\times \Delta \tilde{B}_{j}(k)\right) w(k)+\left(\Lambda_{\ell(k)}(k) \mathcal{C}_{j}+\bar{\beta} \Lambda_{\ell(k)}(k)\right. \\
& \left.\times \Delta \mathcal{C}_{j}(k)+\tilde{\beta}(k) \Lambda_{\ell(k)}(k) \Delta \mathcal{C}_{j}(k)\right) \chi(k) \\
& +\left(\Lambda_{\ell(k)}(k) \mathcal{D}_{j}+\bar{\beta} \Lambda_{\ell(k)}(k) \Delta \mathcal{D}_{j}(k)\right. \\
& \left.\left.+\tilde{\beta}(k) \Lambda_{\ell(k)}(k) \Delta \mathcal{D}_{j}(k)\right) \tilde{w}(k)\right] \\
e(k)= & \sum_{i, j=1}^{r} h_{i} h_{j} \bar{E}_{i j} \xi(k)
\end{aligned}\right.
$$

where

$$
\begin{aligned}
& \xi(k)=\left[\begin{array}{ll}
x^{T}(k) & x_{f}^{T}(k)
\end{array}\right]^{T}, \quad e(k)=z(k)-z_{f}(k), \\
& \tilde{w}(k)=\left[\begin{array}{llll}
w^{T}(k-1) & w^{T}(k-2) & \cdots & w^{T}(k-L)
\end{array}\right]^{T}, \\
& \chi(k)=\left[\begin{array}{llll}
\xi^{T}(k-1) & \xi^{T}(k-2) & \cdots & \xi^{T}(k-L)
\end{array}\right]^{T}, \\
& \tilde{\alpha}(k)=\alpha(k)-\bar{\alpha}, \quad \tilde{\beta}(k)=\beta(k)-\bar{\beta}, \\
& \Delta \tilde{A}_{j}(k)=\operatorname{diag}\left\{0, \Delta A_{f j}(k)\right\} \text {, } \\
& \bar{A}_{i j}=\left[\begin{array}{cc}
A_{i} & 0 \\
0 & A_{f j}
\end{array}\right], \tilde{A}_{j}=\left[\begin{array}{cc}
0 & 0 \\
B_{f j} C & 0
\end{array}\right], \\
& \Delta \tilde{A}_{j}(k)=\left[\begin{array}{cc}
0 & 0 \\
\Delta B_{f j}(k) C & 0
\end{array}\right] \text {, } \\
& \bar{B}_{i}=\left[\begin{array}{c}
B_{i} \\
0
\end{array}\right], \tilde{B}_{j}=\left[\begin{array}{ll}
0 & D^{T} B_{f j}^{T}
\end{array}\right]^{T}, \\
& \Delta \tilde{B}_{j}(k)=\left[\begin{array}{ll}
0 & D^{T} \Delta B_{f j}^{T}(k)
\end{array}\right]^{T}, \\
& \mathcal{C}_{j}=\operatorname{diag}\{\underbrace{\tilde{A}_{j}, \cdots, \tilde{A}_{j}}_{L}\}, \\
& \Delta \mathcal{C}_{j}(k)=\operatorname{diag}\{\underbrace{\Delta \tilde{A}_{j}(k), \cdots, \Delta \tilde{A}_{j}(k)}_{L}\}, \\
& \mathcal{D}_{j}=\operatorname{diag}\{\underbrace{\tilde{B}_{j}, \cdots, \tilde{B}_{j}}_{L}\}, \\
& \Delta \mathcal{D}_{j}(k)=\operatorname{diag}\{\underbrace{\Delta \tilde{B}_{j}(k), \cdots, \Delta \tilde{B}_{j}(k)}_{L}\}, \\
& \bar{E}_{i j}=\left[\begin{array}{ll}
E_{i} & -E_{j}
\end{array}\right], \quad \tilde{G}_{i}=\operatorname{diag}\left\{G_{i}, 0\right\},
\end{aligned}
$$

$$
\begin{aligned}
\bar{\Lambda}_{\ell(k)}(k)= & {[\underbrace{p_{1}(k) I, \cdots, p_{\ell(k)}(k) I, 0, \cdots, 0}_{L}], } \\
\tilde{\Lambda}_{\ell(k)}(k)= & {[\underbrace{\left(\lambda_{1}(k)-p_{1}(k)\right) I, \cdots,\left(\lambda_{\ell(k)}(k)-p_{\ell(k)}(k)\right) I}_{\ell(k)},} \\
\underbrace{0, \cdots, 0}_{L-\ell(k)}], & \bar{\Lambda}_{\ell(k)}(k)+\tilde{\Lambda}_{\ell(k)}(k) .
\end{aligned}
$$

It is clear that

$$
\begin{aligned}
& \mathbb{E}\{\tilde{\alpha}(k)\}=0, \mathbb{E}\left\{\tilde{\alpha}^{2}(k)\right\}:=\alpha^{*}=\bar{\alpha}(1-\bar{\alpha}), \\
& \mathbb{E}\{\tilde{\beta}(k)\}=0, \mathbb{E}\left\{\tilde{\beta}^{2}(k)\right\}:=\beta^{*}=\bar{\beta}(1-\bar{\beta}) .
\end{aligned}
$$

Before proceeding further, we first introduce the following definition.

Definition 1: [14] For the filtering error dynamics (11) and every initial conditions $\phi$, the trivial solution is said to be exponentially mean-square stable if, in the case of $w(k)=0$, there exist constants $\delta>0$ and $0<\kappa<1$ such that

$$
\mathbb{E}\left\{\|\xi(k)\|^{2}\right\} \leq \delta \kappa^{k} \sup _{s \in \mathcal{S}} \mathbb{E}\left\{\|\phi(s)\|^{2}\right\}, \quad \forall k \geq 0 .
$$

The purpose of this paper is to design a non-fragile $H_{\infty}$ fil(11) ter in the form of (10) such that the filtering error dynamic (11) is exponentially mean-square stable and the $H_{\infty}$ performance is satisfied. More specifically, we wish that the following two requirements are satisfied simultaneously:

$R 1$ ): The filtering error dynamics (11) is exponentially mean-square stable;

$R 2)$ : Under zero-initial condition, the estimation error $e(k)$ satisfies

$$
\sum_{k=0}^{\infty} \mathbb{E}\left\{\|e(k)\|^{2}\right\} \leq \gamma^{2} \sum_{k=0}^{\infty}\|v(k)\|^{2}
$$

for all nonzero $v(k)=\left[\begin{array}{ll}w^{T}(k) & \tilde{w}^{T}(k)\end{array}\right]$, where $\gamma>0$ is a prescribed scalar.

\section{MAIN RESULTS}

First, we give the following lemmas that will be used in the proofs of our main results in this paper.

Lemma 1: [52] Let $L=L^{T}, H, M$ and $N$ be real matrices of appropriate dimensions with $M$ satisfying $M M^{T} \leq I$. Then, $L+H M N+N^{T} M^{T} H^{T}<0$, if and only if there exists a positive scalar $\varepsilon>0$ such that $L+\varepsilon^{-1} H H^{T}+\varepsilon N^{T} N<0$ or equivalently

$$
\left[\begin{array}{ccc}
L & H & \varepsilon N^{T} \\
H^{T} & -\varepsilon I & 0 \\
\varepsilon N & 0 & -\varepsilon I
\end{array}\right]<0
$$

Lemma 2: [20] For any real matrices $X_{i j}$ for $i, j=$ $1,2, \cdots, r$ and $r>0$ with appropriate dimensions, we have

$$
\sum_{i=1}^{r} \sum_{j=1}^{r} \sum_{s=1}^{r} \sum_{t=1}^{r} h_{i} h_{j} h_{s} h_{t} X_{i j}^{T} S X_{s t} \leqslant \sum_{i=1}^{r} \sum_{j=1}^{r} h_{i} h_{j} X_{i j}^{T} S X_{i j}
$$

where $S$ is a symmetric positive-definite matrix with appropriate dimensions.

Now, we have the following analysis result that serves as a theoretical basis for the subsequent design problem. 
Theorem 1: Let the filter parameters of the nominal fuzzy system (10) (with $\Delta A_{f i}(k)=0$ and $\Delta B_{f i}(k)=0$ ) and a prescribed $H_{\infty}$ performance $\gamma>0$ be given. Then, the nominal fuzzy system of (11) (with $\Delta A_{f i}(k)=0$ and $\left.\Delta B_{f i}(k)=0\right)$ is exponentially stable with disturbance attenuation level $\gamma$ if there exist matrices $P>0, Q>0$ and $R_{l}>0(l=1,2, \cdots, L)$ such that the following matrix inequalities hold for all $i, j=1,2, \ldots, r$ :

$$
\begin{aligned}
& \mathcal{Z}_{i i}^{T} \tilde{P} \mathcal{Z}_{i i}+\hat{\mathcal{Z}}_{i i}^{T} \overline{\mathcal{P}} \hat{\mathcal{Z}}_{i i}+\bar{P}<0, \\
& \left(\mathcal{Z}_{i j}+\mathcal{Z}_{j i}\right)^{T} \tilde{P}\left(\mathcal{Z}_{i j}+\mathcal{Z}_{j i}\right)+\left(\hat{\mathcal{Z}}_{i j}+\hat{\mathcal{Z}}_{j i}\right)^{T} \overline{\mathcal{P}} \\
& \quad \times\left(\hat{\mathcal{Z}}_{i j}+\hat{\mathcal{Z}}_{j i}\right)+4 \bar{P}<0,
\end{aligned}
$$

where

$$
\begin{aligned}
& \mathcal{Z}_{i j}=\left[\begin{array}{ccc}
\bar{A}_{i j}+m_{0} \tilde{A}_{j} & \tilde{G}_{i} & \Lambda_{\ell(k)}^{m} \mathcal{C}_{j} \\
\bar{E}_{i j} & 0 & 0
\end{array}\right. \\
& \left.\begin{array}{cc}
\bar{B}_{i}+m_{0} \tilde{B}_{j} & \Lambda_{\ell(k)}^{m} \mathcal{D}_{j} \\
0 & 0
\end{array}\right], \\
& \tilde{P}=\operatorname{diag}\{P, I\}, \mathcal{P}=I_{L+3} \otimes P, \\
& \tilde{\mathcal{Z}}_{1 i j}=\left[\begin{array}{ccccc}
f_{0} \bar{A}_{i j} & 0 & 0 & 0 & 0 \\
0 & 0 & 0 & f_{0} \bar{B}_{i} & 0
\end{array}\right], \\
& \tilde{\mathcal{Z}}_{2 i j}=\operatorname{diag}\left\{g_{0} \tilde{A}_{j}, f_{0} \tilde{G}_{i}, \mathcal{G}_{L} \mathcal{C}_{j}, g_{0} \tilde{B}_{j}, \mathcal{G}_{L} \mathcal{D}_{j}\right\}, \\
& \hat{\mathcal{Z}}_{i j}=\left[\tilde{\mathcal{Z}}_{1 i j}^{T}, \tilde{\mathcal{Z}}_{2 i j}^{T}\right]^{T}, \hat{P}=\operatorname{diag}\left\{\bar{Q},-Q,-\mathcal{R}_{L}\right\} \text {, } \\
& \mathcal{R}_{L}=\operatorname{diag}\left\{R_{1}, \cdots, R_{L}\right\}, \tilde{P}=\operatorname{diag}\left\{\hat{P},-\gamma^{2} I,-\gamma^{2} I_{L}\right\}, \\
& \bar{Q}=-P+\left(\tau_{M}-\tau_{m}+1\right) Q+\sum_{l=1}^{L} R_{l}, \overline{\mathcal{P}}=I_{2 L+5} \otimes P, \\
& m_{l}=\frac{\bar{p}_{l}+\underline{p} l}{2}, d_{l}=\frac{\bar{p}_{l}-\underline{p}}{2}, \\
& \Lambda_{\ell(k)}^{m}=[\underbrace{m_{1} I, \cdots, m_{\ell(k)} I, 0, \cdots, 0}_{L}], \\
& \Lambda_{\ell(k)}^{d}=[\underbrace{d_{1} I, \cdots, d_{\ell(k)} I, 0, \cdots, 0}_{L}], \\
& \bar{\Lambda}_{\ell(k)}=[\underbrace{\left.\bar{p}_{1} I, \cdots, \bar{p}_{\ell(k)} I, 0, \cdots, 0\right]}_{L}, f_{0}=\sqrt{4 d_{0}+4 \operatorname{tr}\left(\Lambda_{\ell(k)}^{d}\right)} \text {, } \\
& g_{0}=\sqrt{\bar{q}_{0}+\bar{p}_{0}^{2}-m_{0}^{2}+4 d_{0}+4 \operatorname{tr}\left(\Lambda_{\ell(k)}^{d}\right)+4 d_{0} \operatorname{tr}\left(\Lambda_{\ell(k)}^{m}\right)}, \\
& g_{l}=\sqrt{8 d_{l}+4 d_{0} m_{l}+\bar{p}_{l}^{2}+2 \bar{q}_{l}-m_{l}^{2}}, \\
& \mathcal{G}_{L}=\operatorname{diag}\{\underbrace{g_{1} I, \cdots, g_{\ell(k)} I, 0, \cdots, 0}_{L}\} .
\end{aligned}
$$

Proof: First, for a positive definite matrix $P$ satisfying equations (16) and (17), it follows that

$$
\begin{aligned}
& \mathbb{E}\left\{\tilde{\Lambda}_{\ell(k)}^{T}(k) P \tilde{\Lambda}_{\ell(k)}(k)\right\} \\
=\quad & \mathbb{E}\left\{\left[\left(\lambda_{1}(k)-p_{1}(k)\right) I, \cdots,\left(\lambda_{\ell(k)}(k)-p_{\ell(k)}(k)\right) I,\right.\right. \\
& 0, \cdots, 0]^{T} P\left[\left(\lambda_{1}(k)-p_{1}(k)\right) I, \cdots,\right. \\
& \left.\left.\left(\lambda_{\ell(k)}(k)-p_{\ell(k)}(k)\right) I, 0, \cdots, 0\right]\right\} \\
= & \operatorname{diag}\{\underbrace{q_{1}(k) P, \cdots, q_{\ell(k)}(k) P, 0, \cdots, 0}_{L}\} \\
= & \Lambda_{\ell(k)}^{*}(k) \mathcal{P}_{L},
\end{aligned}
$$

where $\Lambda_{\ell(k)}^{*}(k)=\operatorname{diag}\{\underbrace{q_{1}(k) I, \cdots, q_{\ell(k)}(k) I, 0, \cdots, 0}_{L}\}$,

$\mathcal{P}_{L}=I_{L} \otimes P$.

Next, in order to show that the nominal fuzzy system of (11) (with $\Delta A_{f i}(k)=0$ and $\Delta B_{f i}(k)=0$ ) is exponentially stable with disturbance attenuation level $\gamma$, we choose the following Lyapunov functional:

$$
V(k)=\sum_{i=1}^{4} V_{i}(k)
$$

where

$$
\begin{aligned}
& V_{1}(k)=\xi^{T}(k) P \xi(k), V_{2}(k)=\sum_{i=k-\tau(k)}^{k-1} \xi^{T}(i) Q \xi(i), \\
& V_{3}(k)=\sum_{n=k-\tau_{M}+1}^{k-\tau_{m}} \sum_{i=n}^{k-1} \xi^{T}(i) Q \xi(i), \\
& V_{4}(k)=\sum_{l=1}^{L} \sum_{i=k-l}^{k-1} x^{T}(i) R_{l} x(i) .
\end{aligned}
$$

Then, it can be obtained from (11) that

$$
\begin{aligned}
& \mathbb{E}\left\{\Delta V_{1}(k)\right\} \\
& =\mathbb{E}\left\{\xi^{T}(k+1) P \xi(k+1)-\xi^{T}(k) P \xi(k)\right\} \\
& =\mathbb{E}\left\{\sum _ { i , j , s , t = 1 } ^ { r } h _ { i } h _ { j } h _ { s } h _ { t } \left[\left(\bar{A}_{i j}+\bar{\alpha} \Delta \tilde{A}_{j}(k)\right.\right.\right. \\
& +\tilde{\alpha}(k) \Delta \tilde{A}_{j}(k)+\lambda_{0}(k) \tilde{A}_{j}+\bar{\beta} \lambda_{0}(k) \Delta \tilde{A}_{j}(k) \\
& \left.+\tilde{\beta}(k) \lambda_{0}(k) \Delta \tilde{A}_{j}(k)\right) \xi(k)+\tilde{G}_{i} \xi(k-\tau(k)) \\
& +\left(\bar{B}_{i}+\lambda_{0}(k) \tilde{B}_{j}+\bar{\beta} \lambda_{0}(k) \Delta \tilde{B}_{j}(k)+\tilde{\beta}(k) \lambda_{0}(k)\right. \\
& \left.\times \Delta \tilde{B}_{j}(k)\right) w(k)+\left(\Lambda_{\ell(k)}(k) \mathcal{C}_{j}+\bar{\beta} \Lambda_{\ell(k)}(k) \Delta \mathcal{C}_{j}(k)\right. \\
& \left.+\tilde{\beta}(k) \Lambda_{\ell(k)}(k) \Delta \mathcal{C}_{j}(k)\right) \chi(k)+\left(\Lambda_{\ell(k)}(k) \mathcal{D}_{j}\right. \\
& \left.\left.+\bar{\beta} \Lambda_{\ell(k)}(k) \Delta \mathcal{D}_{j}(k)+\tilde{\beta}(k) \Lambda_{\ell(k)}(k) \Delta \mathcal{D}_{j}(k)\right) \tilde{w}(k)\right]^{T} \\
& \times P\left[\left(\bar{A}_{s t}+\bar{\alpha} \Delta \tilde{A}_{t}(k)+\tilde{\alpha}(k) \Delta \tilde{A}_{t}(k)+\lambda_{0}(k) \tilde{A}_{t}\right.\right. \\
& \left.+\bar{\beta} \lambda_{0}(k) \Delta \tilde{A}_{t}(k)+\tilde{\beta}(k) \lambda_{0}(k) \Delta \tilde{A}_{t}(k)\right) \xi(k) \\
& +\tilde{G}_{s} \xi(k-\tau(k))+\left(\bar{B}_{s}+\lambda_{0}(k) \tilde{B}_{t}+\bar{\beta} \lambda_{0}(k)\right. \\
& \left.\times \Delta \tilde{B}_{t}(k)+\tilde{\beta}(k) \lambda_{0}(k) \Delta \tilde{B}_{t}(k)\right) w(k)+\left(\Lambda_{\ell(k)}(k) \mathcal{C}_{t}\right. \\
& \left.+\bar{\beta} \Lambda_{\ell(k)}(k) \Delta \mathcal{C}_{t}(k)+\tilde{\beta}(k) \Lambda_{\ell(k)}(k) \Delta \mathcal{C}_{t}(k)\right) \chi(k) \\
& +\left(\Lambda_{\ell(k)}(k) \mathcal{D}_{t} \tilde{w}(k)+\bar{\beta} \Lambda_{\ell(k)}(k) \Delta \mathcal{D}_{t}(k)\right. \\
& \left.\left.\left.+\tilde{\beta}(k) \Lambda_{\ell(k)}(k) \Delta \mathcal{D}_{t}(k)\right) \tilde{w}(k)\right]-\xi^{T}(k) P \xi(k)\right\} \\
& =\mathbb{E}\left\{\sum _ { i , j , s , t = 1 } ^ { r } h _ { i } h _ { j } h _ { s } h _ { t } \left[\xi ^ { T } ( k ) \left(\left(\bar{A}_{i j}+\bar{\alpha} \Delta \tilde{A}_{j}(k)\right.\right.\right.\right. \\
& +\tilde{\alpha}(k) \Delta \tilde{A}_{j}(k)+\lambda_{0}(k) \tilde{A}_{j}+\bar{\beta} \lambda_{0}(k) \Delta \tilde{A}_{j}(k) \\
& \left.+\tilde{\beta}(k) \lambda_{0}(k) \Delta \tilde{A}_{j}(k)\right)^{T} P\left(\bar{A}_{s t}+\bar{\alpha} \Delta \tilde{A}_{t}(k)\right. \\
& +\tilde{\alpha}(k) \Delta \tilde{A}_{t}(k)+\lambda_{0}(k) \tilde{A}_{t}+\bar{\beta} \lambda_{0}(k) \Delta \tilde{A}_{t}(k) \\
& \left.\left.+\tilde{\beta}(k) \lambda_{0}(k) \Delta \tilde{A}_{t}(k)\right)-P\right) \xi(k)+2 \xi^{T}(k)\left(\bar{A}_{i j}\right. \\
& +\bar{\alpha} \Delta \tilde{A}_{i}(k)+\tilde{\alpha}(k) \Delta \tilde{A}_{j}(k)+\lambda_{0}(k) \tilde{A}_{j} \\
& \left.+\bar{\beta} \lambda_{0}(k) \Delta \tilde{A}_{j}(k)+\tilde{\beta}(k) \lambda_{0}(k) \Delta \tilde{A}_{j}(k)\right)^{T} P \\
& \times \tilde{G}_{s} \xi(k-\tau(k))+2 \xi^{T}(k)\left(\bar{A}_{i j}+\bar{\alpha} \Delta \tilde{A}_{j}(k)\right. \\
& +\tilde{\alpha}(k) \Delta \tilde{A}_{j}(k)+\lambda_{0}(k) \tilde{A}_{j}+\bar{\beta} \lambda_{0}(k) \Delta \tilde{A}_{j}(k) \\
& \left.+\tilde{\beta}(k) \lambda_{0}(k) \Delta \tilde{A}_{j}(k)\right)^{T} P\left(\bar{B}_{s}+\lambda_{0}(k) \tilde{B}_{t}\right. \\
& \left.+\bar{\beta} \lambda_{0}(k) \Delta \tilde{B}_{t}(k)+\tilde{\beta}(k) \lambda_{0}(k) \Delta \tilde{B}_{t}(k)\right) w(k) \\
& +2 \xi^{T}(k)\left(\bar{A}_{i j}+\bar{\alpha} \Delta \tilde{A}_{j}(k)+\tilde{\alpha}(k) \Delta \tilde{A}_{j}(k)\right.
\end{aligned}
$$




$$
\begin{aligned}
& +\lambda_{0}(k) \tilde{A}_{j}+\bar{\beta} \lambda_{0}(k) \Delta \tilde{A}_{j}(k)+\tilde{\beta}(k) \lambda_{0}(k) \\
& \left.\times \Delta \tilde{A}_{j}(k)\right)^{T} P\left(\Lambda_{\ell(k)}(k) \mathcal{C}_{t}+\bar{\beta} \Lambda_{\ell(k)}(k) \Delta \mathcal{C}_{t}(k)\right. \\
& \left.+\tilde{\beta}(k) \Lambda_{\ell(k)}(k) \Delta \mathcal{C}_{t}(k)\right) \chi(k)+2 \xi^{T}(k)\left(\bar{A}_{i j}\right. \\
& +\bar{\alpha} \Delta \tilde{A}_{j}(k)+\tilde{\alpha}(k) \Delta \tilde{A}_{j}(k)+\lambda_{0}(k) \tilde{A}_{j} \\
& \left.+\bar{\beta} \lambda_{0}(k) \Delta \tilde{A}_{j}(k)+\tilde{\beta}(k) \lambda_{0}(k) \Delta \tilde{A}_{j}(k)\right)^{T} P \\
& \times\left(\tilde{\beta}(k) \Lambda_{\ell(k)}(k) \Delta \mathcal{D}_{t}(k)+\bar{\beta} \Lambda_{\ell(k)}(k) \Delta \mathcal{D}_{t}(k)\right. \\
& \left.+\Lambda_{\ell(k)}(k) \mathcal{D}_{t}\right) \tilde{w}(k)+\xi^{T}(k-\tau(k)) \tilde{G}_{i}^{T} P \tilde{G}_{s} \\
& \times \xi(k-\tau(k))+2 \xi^{T}(k-\tau(k)) \tilde{G}_{i}^{T} P\left(\lambda_{0}(k) \tilde{B}_{t}\right. \\
& \left.+\bar{B}_{s}+\bar{\beta} \lambda_{0}(k) \Delta \tilde{B}_{t}(k)+\tilde{\beta}(k) \lambda_{0}(k) \Delta \tilde{B}_{t}(k)\right) w(k) \\
& +2 \xi^{T}(k-\tau(k)) \tilde{G}_{i}^{T} P\left(\Lambda_{\ell(k)}(k) \mathcal{C}_{t}\right. \\
& \left.+\bar{\beta} \Lambda_{\ell(k)}(k) \Delta \mathcal{C}_{t}(k)+\tilde{\beta}(k) \Lambda_{\ell(k)}(k) \Delta \mathcal{C}_{t}(k)\right) \chi(k) \\
& +2 \xi^{T}(k-\tau(k)) \tilde{G}_{i}^{T} P\left(\Lambda_{\ell(k)}(k) \mathcal{D}_{t}\right. \\
& +\bar{\beta} \Lambda_{\ell(k)}(k) \Delta \mathcal{D}_{t}(k)+\tilde{\beta}(k) \Lambda_{\ell(k)}(k) \\
& \left.\times \Delta \mathcal{D}_{t}(k)\right) \tilde{w}(k)+w^{T}(k)\left(\bar{B}_{i}+\lambda_{0}(k) \tilde{B}_{j}\right. \\
& \left.+\bar{\beta} \lambda_{0}(k) \Delta \tilde{B}_{j}(k)+\tilde{\beta}(k) \lambda_{0}(k) \Delta \tilde{B}_{j}(k)\right)^{T} \\
& \times P\left(\bar{B}_{s}+\lambda_{0}(k) \tilde{B}_{t}+\bar{\beta} \lambda_{0}(k) \Delta \tilde{B}_{t}(k)\right. \\
& \left.+\tilde{\beta}(k) \lambda_{0}(k) \Delta \tilde{B}_{t}(k)\right) w(k)+2 w^{T}(k)\left(\bar{B}_{i}\right. \\
& \left.+\lambda_{0}(k) \tilde{B}_{j}+\bar{\beta} \lambda_{0}(k) \Delta \tilde{B}_{j}(k)+\tilde{\beta}(k) \lambda_{0}(k) \Delta \tilde{B}_{j}(k)\right)^{T} \\
& \times P\left(\Lambda_{\ell(k)}(k) \mathcal{C}_{t}+\tilde{\beta}(k) \Lambda_{\ell(k)}(k) \Delta \mathcal{C}_{t}(k)\right. \\
& \left.+\bar{\beta} \Lambda_{\ell(k)}(k) \Delta \mathcal{C}_{t}(k)\right) \chi(k)+2 w^{T}(k)\left(\bar{B}_{i}+\lambda_{0}(k) \tilde{B}_{j}\right. \\
& \left.+\bar{\beta} \lambda_{0}(k) \Delta \tilde{B}_{j}(k)+\tilde{\beta}(k) \lambda_{0}(k) \Delta \tilde{B}_{j}(k)\right)^{T} \\
& \times P\left(\Lambda_{\ell(k)}(k) \mathcal{D}_{t}+\bar{\beta} \Lambda_{\ell(k)}(k) \Delta \mathcal{D}_{t}(k)\right. \\
& \left.+\tilde{\beta}(k) \Lambda_{\ell(k)}(k) \Delta \mathcal{D}_{t}(k)\right) \tilde{w}(k)+\chi^{T}(k)\left(\Lambda_{\ell(k)}(k) \mathcal{C}_{j}\right. \\
& \left.+\bar{\beta} \Lambda_{\ell(k)}(k) \Delta \mathcal{C}_{j}(k)+\tilde{\beta}(k) \Lambda_{\ell(k)}(k) \Delta \mathcal{C}_{j}(k)\right)^{T} P \\
& \times\left(\Lambda_{\ell(k)}(k) \mathcal{C}_{t}+\bar{\beta} \Lambda_{\ell(k)}(k) \Delta \mathcal{C}_{t}(k)\right. \\
& \left.+\tilde{\beta}(k) \Lambda_{\ell(k)}(k) \Delta \mathcal{C}_{t}(k)\right) \chi(k)+2 \chi^{T}(k)\left(\Lambda_{\ell(k)}(k) \mathcal{C}_{j}\right. \\
& \left.+\bar{\beta} \Lambda_{\ell(k)}(k) \Delta \mathcal{C}_{j}(k)+\tilde{\beta}(k) \Lambda_{\ell(k)}(k) \Delta \mathcal{C}_{j}(k)\right)^{T} \\
& \times P\left(\Lambda_{\ell(k)}(k) \mathcal{D}_{t}+\bar{\beta} \Lambda_{\ell(k)}(k) \Delta \mathcal{D}_{t}(k)\right. \\
& \left.+\tilde{\beta}(k) \Lambda_{\ell(k)}(k) \Delta \mathcal{D}_{t}(k)\right) \tilde{w}(k)+\tilde{w}^{T}(k)\left(\Lambda_{\ell(k)}(k) \mathcal{D}_{j}\right. \\
& \left.+\bar{\beta} \Lambda_{\ell(k)}(k) \Delta \mathcal{D}_{j}(k)+\tilde{\beta}(k) \Lambda_{\ell(k)}(k) \Delta \mathcal{D}_{j}(k)\right)^{T} P \\
& \times\left(\Lambda_{\ell(k)}(k) \mathcal{D}_{t}+\bar{\beta} \Lambda_{\ell(k)}(k) \Delta \mathcal{D}_{t}(k)\right. \\
& \left.\left.\left.+\tilde{\beta}(k) \Lambda_{\ell(k)}(k) \Delta \mathcal{D}_{t}(k)\right) \tilde{w}(k)\right]\right\}
\end{aligned}
$$

Moreover, taking the elementary inequality $2 a b \leq a^{2}+b^{2}$ into consideration, we have

$$
\begin{aligned}
& \mathbb{E}\left\{\Delta V_{1}(k)\right\} \leq \\
& \quad \sum_{i, j, s, t=1}^{r} h_{i} h_{j} h_{s} h_{t}\left[\xi ^ { T } ( k ) \left(\left(1+4 d_{0}+4 \operatorname{tr}\left(\Lambda_{\ell(k)}^{d}\right)\right) \bar{A}_{i j}^{T} P\right.\right. \\
& \times \bar{A}_{s t}+2 \bar{\alpha} \bar{A}_{i j}^{T} P \Delta \tilde{A}_{t}(k)+2 m_{0} \bar{A}_{i j}^{T} P \tilde{A}_{t}+2 \bar{\beta} m_{0} \bar{A}_{i j}^{T} P \\
& \times \Delta \tilde{A}_{t}(k)+\bar{\alpha}^{2}\left(1+4 d_{0}+4 \operatorname{tr}\left(\Lambda_{\ell(k)}^{d}\right)\right) \Delta \tilde{A}_{j}^{T}(k) P \Delta \tilde{A}_{t}(k) \\
& +2 \bar{\alpha} m_{0} \Delta \tilde{A}_{j}^{T}(k) P \tilde{A}_{t}+2 \bar{\alpha} m_{0} \Delta \tilde{A}_{j}^{T}(k) P \Delta \tilde{A}_{t}(k) \\
& +\alpha^{*} \Delta \tilde{A}_{j}^{T}(k) P \Delta \tilde{A}_{t}(k)+\left(\bar{q}_{0}+\bar{p}_{0}^{2}+4 d_{0}+4\left(\operatorname{tr}\left(\Lambda_{\ell(k)}^{d}\right)\right.\right. \\
& \left.\left.+d_{0} \operatorname{tr}\left(\Lambda_{\ell(k)}^{m}\right)\right)\right) \tilde{A}_{j}^{T} P \tilde{A}_{t}+2 \bar{\beta}\left(\bar{q}_{0}+\bar{p}_{0}^{2}\right) \tilde{A}_{j}^{T} P \Delta \tilde{A}_{t}(k) \\
& +\bar{\beta}^{2}\left(\bar{q}_{0}+\bar{p}_{0}^{2}+4 d_{0}+4\left(\operatorname{tr}\left(\Lambda_{\ell(k)}^{d}\right)\right.\right. \\
& \left.+d_{0} \operatorname{tr}\left(\Lambda_{\ell(k)}^{m}\right)\right) \Delta \tilde{A}_{j}^{T}(k) P \Delta \tilde{A}_{t}(k) \\
& +\beta^{*}\left(\bar{q}_{0}+\bar{p}_{0}^{2}+2\left(\operatorname{tr}\left(\Lambda_{\ell(k)}^{d}\right)+d_{0} \operatorname{tr}\left(\Lambda_{\ell(k)}^{m}\right)\right)\right) \\
& \left.\times \Delta \tilde{A}_{j}^{T}(k) P \Delta \tilde{A}_{t}(k)-P\right) \xi(k)+2 \xi^{T}(k)\left(\bar{A}_{i j}^{T} P \tilde{G}_{s}\right. \\
& \left.+\bar{\alpha} \Delta \tilde{A}_{j}^{T}(k) P \tilde{G}_{s}+\bar{\beta}_{0} m_{0} \Delta \tilde{A}_{j}^{T}(k) P \tilde{G}_{s}\right) \\
& \times \xi(k-\tau(k))+m_{0} \tilde{A}_{j}^{T} P \tilde{G}_{s}+2 \xi{ }^{T}(k)\left(\bar{A}_{i j}^{T} P \bar{B}_{s}\right. \\
& +m_{0} \bar{A}_{i j}^{T} P \tilde{B}_{t}+\bar{\beta} m_{0} \bar{A}_{i j}^{T} P \Delta \tilde{B}_{t}(k) \\
& +\bar{\alpha} \Delta \tilde{A}_{j}^{T}(k) P \bar{B}_{s}+\bar{\alpha} m_{0} \Delta \tilde{A}_{j}^{T}(k) P \tilde{B}_{t} \\
& +\bar{\alpha} \bar{\beta} m_{0} \Delta \tilde{A}_{j}^{T}(k) P \Delta \tilde{B}_{t}(k)+\bar{\beta}_{0} \Delta \tilde{A}_{j}^{T}(k) P \bar{B}_{s} \\
& \quad
\end{aligned}
$$

$$
\begin{aligned}
& +\bar{\beta}\left(\bar{q}_{0}+\bar{p}_{0}^{2}\right) \tilde{A}_{j}^{T} P \Delta \tilde{B}_{t}(k)+\left(\bar{q}_{0}+\bar{p}_{0}^{2}\right) \tilde{A}_{j}^{T} P \tilde{B}_{t} \\
& +\bar{\beta}\left(\bar{q}_{0}+\bar{p}_{0}^{2}\right) \Delta \tilde{A}_{j}^{T}(k) P \tilde{B}_{t}+m_{0} \tilde{A}_{j}^{T} P \bar{B}_{s} \\
& +\bar{\beta}^{2}\left(\bar{q}_{0}+\bar{p}_{0}^{2}\right) \Delta \tilde{A}_{j}^{T}(k) P \Delta \tilde{B}_{t}(k)+\beta^{*}\left(\bar{q}_{0}+\bar{p}_{0}^{2}\right) \\
& \left.\times \Delta \tilde{A}_{j}^{T}(k) P \Delta \tilde{B}_{t}(k)\right) w(k)+2 \xi^{T}(k)\left(\bar{A}_{i j}^{T} P \Lambda_{\ell(k)}^{m} \mathcal{C}_{t}\right. \\
& +\bar{A}_{i j}^{T} P \bar{\beta} \Lambda_{\ell(k)}^{m} \Delta \mathcal{C}_{t}(k)+\bar{\alpha} \Delta \tilde{A}_{j}^{T}(k) P \Lambda_{\ell(k)}^{m} \mathcal{C}_{t} \\
& +\bar{\alpha} \bar{\beta} \Delta \tilde{A}_{j}^{T}(k) P \Lambda_{\ell(k)}^{m} \Delta \mathcal{C}_{t}(k)+\tilde{A}_{j}^{T} P m_{0} \Lambda_{\ell(k)}^{m} \mathcal{C}_{t} \\
& +\bar{\beta} \tilde{A}_{j}^{T} P m_{0} \Lambda_{\ell(k)}^{m} \Delta \mathcal{C}_{t}(k)+\bar{\beta} \Delta \tilde{A}_{j}^{T}(k) P m_{0} \Lambda_{\ell(k)}^{m} \mathcal{C}_{t} \\
& +\bar{\beta}^{2} \Delta \tilde{A}_{j}^{T}(k) P m_{0} \Lambda_{\ell(k)}^{m} \Delta \mathcal{C}_{t}(k)+\beta^{*} \Delta \tilde{A}_{j}^{T}(k) P \\
& \left.\times m_{0} \Lambda_{\ell(k)}^{m} \Delta \mathcal{C}_{t}(k)\right) \chi(k)+2 \xi^{T}(k)\left(\bar{A}_{i j}^{T} P \Lambda_{\ell(k)}^{m} \mathcal{D}_{t}\right. \\
& +\bar{A}_{i j}^{T} P \bar{\beta} \Lambda_{\ell(k)}^{m} \Delta \mathcal{D}_{t}(k)+\bar{\alpha} \bar{\beta} \Delta \tilde{A}_{j}^{T}(k) P \Lambda_{\ell(k)}^{m} \Delta \mathcal{D}_{t}(k) \\
& +\bar{\alpha} \Delta \tilde{A}_{j}^{T}(k) P \Lambda_{\ell(k)}^{m} \mathcal{D}_{t}+\bar{\beta} \Delta \tilde{A}_{j}^{T}(k) P \\
& \times m_{0} \Lambda_{\ell(k)}^{m} \mathcal{D}_{t}+\bar{\beta} \tilde{A}_{j}^{T} P m_{0} \Lambda_{\ell(k)}^{m} \Delta \mathcal{D}_{t}(k) \\
& +\beta^{*} \Delta \tilde{A}_{j}^{T}(k) P m_{0} \Lambda_{\ell(k)}^{m} \Delta \mathcal{D}_{t}(k)+\tilde{A}_{j}^{T} P m_{0} \Lambda_{\ell(k)}^{m} \mathcal{D}_{t} \\
& \left.+\bar{\beta}^{2} \Delta \tilde{A}_{j}^{T}(k) P m_{0} \Lambda_{\ell(k)}^{m} \Delta \mathcal{D}_{t}(k)\right) \tilde{w}(k)+\xi^{T}(k-\tau(k)) \\
& \times \tilde{G}_{i}^{T}\left(1+4 d_{0}+4 \operatorname{tr}\left(\Lambda_{\ell(k)}^{d}\right)\right) P \tilde{G}_{s} \xi(k-\tau(k)) \\
& +2 \xi^{T}(k-\tau(k))\left(\tilde{G}_{i}^{T} P \bar{B}_{s}+\bar{\beta} m_{0} \tilde{G}_{i}^{T} P \Delta \tilde{B}_{t}(k)\right. \\
& \left.+m_{0} \tilde{G}_{i}^{T} P \tilde{B}_{t}\right) w(k)+2 \xi^{T}(k-\tau(k))\left(\tilde{G}_{i}^{T} P \Lambda_{\ell(k)}^{m} \mathcal{C}_{t}\right. \\
& \left.+\bar{\beta} \tilde{G}_{i}^{T} P \Lambda_{\ell(k)}^{m} \Delta \mathcal{C}_{t}(k)\right) \chi(k)+2 \xi^{T}(k-\tau(k)) \\
& \times\left(\tilde{G}_{i}^{T} P \Lambda_{\ell(k)}^{m} \mathcal{D}_{t}+\bar{\beta} \tilde{G}_{i}^{T} P \Lambda_{\ell(k)}^{m} \Delta \mathcal{D}_{t}(k)\right) \tilde{w}(k) \\
& +w^{T}(k)\left(\bar{B}_{i}^{T}\left(1+4 d_{0}+4 \operatorname{tr}\left(\Lambda_{\ell}^{d}\right)\right) P \bar{B}_{s}+2 m_{0} \bar{B}_{i}^{T} P \tilde{B}_{t}\right. \\
& +2 \bar{\beta} m_{0} \bar{B}_{i}^{T} P \Delta \tilde{B}_{t}(k)+\left(\bar{q}_{0}+\bar{p}_{0}^{2}+4 d_{0}+4\left(\operatorname{tr}\left(\Lambda_{\ell}^{d}\right)\right.\right. \\
& \left.\left.+d_{0} \operatorname{tr}\left(\Lambda_{\ell}^{m}\right)\right)\right) \tilde{B}_{j}^{T} P \tilde{B}_{t}+2 \bar{\beta}\left(\bar{q}_{0}+\bar{p}_{0}^{2}\right) \tilde{B}_{j}^{T} P \Delta \tilde{B}_{t}(k) \\
& +\bar{\beta}^{2}\left(\bar{q}_{0}+\bar{p}_{0}^{2}+4 d_{0}+4\left(\operatorname{tr}\left(\Lambda_{\ell}^{d}\right)+d_{0} \operatorname{tr}\left(\Lambda_{\ell}^{m}\right)\right)\right) \Delta \tilde{B}_{j}^{T}(k) \\
& \times P \Delta \tilde{B}_{t}(k)+\beta^{*}\left(\bar{q}_{0}+\bar{p}_{0}^{2}+2\left(\operatorname{tr}\left(\Lambda_{\ell}^{d}\right)\right.\right. \\
& \left.\left.\left.+d_{0} \operatorname{tr}\left(\Lambda_{\ell}^{m}\right)\right)\right) \Delta \tilde{B}_{j}^{T}(k) P \Delta \tilde{B}_{t}(k)\right) w(k) \\
& +2 w^{T}(k)\left(\bar{B}_{i}^{T} P \Lambda_{\ell(k)}^{m} \mathcal{C}_{t}+\bar{B}_{i}^{T} P \bar{\beta} \Lambda_{\ell(k)}^{m} \Delta \mathcal{C}_{t}(k)\right. \\
& +\tilde{B}_{j}^{T} P m_{0} \Lambda_{\ell(k)}^{m} \mathcal{C}_{t}+\bar{\beta} \tilde{B}_{j}^{T} P m_{0} \Lambda_{\ell(k)}^{m} \Delta \mathcal{C}_{t}(k) \\
& +\bar{\beta} \Delta \tilde{B}_{j}^{T}(k) P m_{0} \Lambda_{\ell(k)}^{m} \mathcal{C}_{t}+\bar{\beta}^{2} \Delta \tilde{B}_{j}^{T}(k) P m_{0} \Lambda_{\ell(k)}^{m} \\
& \left.\times \Delta \mathcal{C}_{t}(k)+\beta^{*} \Delta \tilde{B}_{j}^{T}(k) P m_{0} \Lambda_{\ell(k)}^{m} \Delta \mathcal{C}_{t}(k)\right) \chi(k) \\
& +2 w^{T}(k)\left(\bar{B}_{i}^{T} P \Lambda_{\ell(k)}^{m} \mathcal{D}_{t}+\tilde{B}_{j}^{T} P m_{0} \Lambda_{\ell(k)}^{m} \mathcal{D}_{t}\right. \\
& +\bar{\beta} \bar{B}_{i}^{T} P \Lambda_{\ell(k)}^{m} \Delta \mathcal{D}_{t}(k)+\bar{\beta} \tilde{B}_{j}^{T} P m_{0} \Lambda_{\ell(k)}^{m} \Delta \mathcal{D}_{t}(k) \\
& +\bar{\beta} \Delta \tilde{B}_{j}^{T}(k) P m_{0} \Lambda_{\ell(k)}^{m} \mathcal{D}_{t}+\bar{\beta}^{2} \Delta \tilde{B}_{j}^{T}(k) P m_{0} \\
& \left.\times \Lambda_{\ell(k)}^{m} \Delta \mathcal{D}_{t}(k)+\beta^{*} \Delta \tilde{B}_{j}^{T}(k) P m_{0} \Lambda_{\ell(k)}^{m} \Delta \mathcal{D}_{t}(k)\right) \tilde{w}(k) \\
& +\chi^{T}(k)\left(\mathcal{C}_{j}^{T}\left(8 \Lambda_{\ell(k)}^{d}+4 d_{0} \Lambda_{\ell(k)}^{m}+\tilde{\Lambda}_{\ell(k)}^{*}\right) \mathcal{P}_{L} \mathcal{C}_{t}\right. \\
& +2 \bar{\beta} \mathcal{C}_{j}^{T} \tilde{\Lambda}_{\ell(k)}^{*} \mathcal{P}_{L} \Delta \mathcal{C}_{t}(k)+\bar{\beta}^{2} \Delta \mathcal{C}_{j}^{T}(k)\left(8 \Lambda_{\ell(k)}^{d}\right. \\
& \left.+4 d_{0} \Lambda_{\ell(k)}^{m}+\tilde{\Lambda}_{\ell(k)}^{*}\right) \mathcal{P}_{L} \Delta \mathcal{C}_{t}(k)+\beta^{*} \Delta \mathcal{C}_{j}^{T}(k)\left(2 \Lambda_{\ell(k)}^{d}\right. \\
& \left.\left.+2 d_{0} \Lambda_{\ell(k)}^{m}+\tilde{\Lambda}_{\ell(k)}^{*}\right) \mathcal{P}_{L} \Delta \mathcal{C}_{t}(k)\right) \chi(k) \\
& +2 \chi^{T}(k)\left(\mathcal{C}_{j}^{T} \tilde{\Lambda}_{\ell(k)}^{*} \mathcal{P}_{L} \mathcal{D}_{t}+\bar{\beta} \mathcal{C}_{j}^{T} \tilde{\Lambda}_{\ell(k)}^{*} \mathcal{P}_{L} \Delta \mathcal{D}_{t}(k)\right. \\
& +\bar{\beta} \Delta \mathcal{C}_{j}^{T}(k) \tilde{\Lambda}_{\ell(k)}^{*} \mathcal{P}_{L} \mathcal{D}_{t}+\bar{\beta}^{2} \Delta \mathcal{C}_{j}^{T}(k) \tilde{\Lambda}_{\ell(k)}^{*} \mathcal{P}_{L} \Delta \mathcal{D}_{t}(k) \\
& \left.+\beta^{*} \Delta \mathcal{C}_{j}^{T}(k) \tilde{\Lambda}_{\ell(k)}^{*} \mathcal{P}_{L} \Delta \mathcal{D}_{t}(k)\right) \tilde{w}(k) \\
& +\tilde{w}^{T}(k)\left(\mathcal{D}_{j}^{T}\left(8 \Lambda_{\ell(k)}^{d}+4 d_{0} \Lambda_{\ell(k)}^{m}+\tilde{\Lambda}_{\ell(k)}^{*}\right) \mathcal{P}_{L} \mathcal{D}_{t}\right. \\
& +2 \bar{\beta} \mathcal{D}_{j}^{T} \tilde{\Lambda}_{\ell(k)}^{*} \mathcal{P}_{L} \Delta \mathcal{D}_{t}(k)+\bar{\beta}^{2} \Delta \mathcal{D}_{j}^{T}(k)\left(8 \Lambda_{\ell(k)}^{d}\right. \\
& \left.+4 d_{0} \Lambda_{\ell(k)}^{m}+\tilde{\Lambda}_{\ell(k)}^{*}\right) \mathcal{P}_{L} \Delta \mathcal{D}_{t}(k)+\beta^{*} \Delta \mathcal{D}_{j}^{T}(k)\left(2 \Lambda_{\ell(k)}^{d}\right. \\
& \left.\left.\left.+2 d_{0} \Lambda_{\ell(k)}^{m}+\tilde{\Lambda}_{\ell(k)}^{*}\right) \mathcal{P}_{L} \Delta \mathcal{D}_{t}(k)\right) \tilde{w}(k)\right]
\end{aligned}
$$

where

$$
\begin{aligned}
& \bar{\Lambda}_{\ell(k)}^{*}=\operatorname{diag}\{\underbrace{\bar{q}_{1} I, \cdots, \bar{q}_{\ell(k)} I, 0, \cdots, 0}_{L}\} \\
& \tilde{\Lambda}_{\ell(k)}^{*}=\bar{\Lambda}_{\ell(k)}^{T} \bar{\Lambda}_{\ell(k)}+\bar{\Lambda}_{\ell(k)}^{*}+\left(1-\delta_{\{i j\}\{s t\}}\right) \bar{\Lambda}_{\ell(k)}^{*}
\end{aligned}
$$


with the kronecker delta function $\delta_{\{i j\}\{s t\}}$ satisfying

$$
\delta_{\{i j\}\{s t\}}= \begin{cases}1, & \text { if } i=s \text { and } j=t \\ 0, & \text { others. }\end{cases}
$$

Also, it can be obtained that

$$
\begin{aligned}
& \mathbb{E}\left\{\Delta V_{2}(k)\right\} \\
& =\mathbb{E}\left\{V_{2}(k+1)-V_{2}(k)\right\} \\
& =\mathbb{E}\left\{\sum_{i=k+1-\tau(k+1)}^{k} \xi^{T}(i) Q \xi(i)-\sum_{i=k-\tau(k)}^{k-1} \xi^{T}(i) Q \xi(i)\right\} \\
& =\mathbb{E}\left\{\xi^{T}(k) Q \xi(k)-\xi^{T}(k-\tau(k)) Q \xi(k-\tau(k))\right. \\
& \left.+\sum_{i=k+1-\tau(k+1)}^{k-1} \xi^{T}(i) Q \xi(i)-\sum_{i=k+1-\tau(k)}^{k-1} \xi^{T}(i) Q \xi(i)\right\} \\
& =\mathbb{E}\left\{\xi^{T}(k) Q \xi(k)-\xi^{T}(k-\tau(k)) Q \xi(k-\tau(k))\right. \\
& +\sum_{i=k+1-\tau(k+1)}^{k-\tau_{m}} \xi^{T}(i) Q \xi(i)+\sum_{i=k+1-\tau_{m}}^{k-1} \xi^{T}(i) Q \xi(i) \\
& \left.-\sum_{i=k+1-\tau(k)}^{k-1} \xi^{T}(i) Q \xi(i)\right\} \\
& \leq \mathbb{E}\left\{\xi^{T}(k) Q \xi(k)-\xi^{T}(k-\tau(k)) Q \xi(k-\tau(k))\right. \\
& \left.+\sum_{i=k+1-\tau_{M}}^{k-\tau_{m}} \xi^{T}(i) Q \xi(i)\right\} \\
& \mathbb{E}\left\{\Delta V_{3}(k)\right\} \\
& =\mathbb{E}\left\{V_{3}(k+1)-V_{3}(k)\right\} \\
& =\mathbb{E}\left\{\sum_{n=k-\tau_{M}+2}^{k+1-\tau_{m}} \sum_{i=n}^{k} \xi^{T}(i) Q \xi(i)\right. \\
& \left.-\sum_{n=k-\tau_{M}+1}^{k-\tau_{m}} \sum_{i=n}^{k-1} \xi^{T}(i) Q \xi(i)\right\} \\
& =\mathbb{E}\left\{\sum_{n=k-\tau_{M}+1}^{k-\tau_{m}} \sum_{i=n+1}^{k} \xi^{T}(i) Q \xi(i)\right. \\
& \left.-\sum_{n=k-\tau_{M}+1}^{k-\tau_{m}} \sum_{i=n}^{k-1} \xi^{T}(i) Q \xi(i)\right\} \\
& =\mathbb{E}\left\{\sum_{n=k-\tau_{M}+1}^{k-\tau_{m}}\left(\xi^{T}(k) Q \xi(k)-\xi^{T}(n) Q \xi(n)\right)\right\} \\
& =\mathbb{E}\left\{\left(\tau_{M}-\tau_{m}\right) \xi^{T}(k) Q \xi(k)\right. \\
& \left.-\sum_{n=k+1-\tau_{M}}^{k-\tau_{m}} \xi^{T}(n) Q \xi(n)\right\}
\end{aligned}
$$

$$
\begin{aligned}
& \mathbb{E}\left\{\Delta V_{4}(k)\right\} \\
= & \mathbb{E}\left\{V_{4}(k+1)-V_{4}(k)\right\} \\
= & \sum_{l=1}^{L}\left\{\sum_{i=k+1-l}^{k} \xi^{T}(i) R_{l} \xi(i)-\sum_{i=k-l}^{k-1} \xi^{T}(i) R_{l} \xi(i)\right\} \\
= & \sum_{l=1}^{L}\left\{\xi^{T}(k) R_{l} \xi(k)-\xi^{T}(k-l) R_{l} \xi(k-l)\right\} .
\end{aligned}
$$

For notational convenience, we denote

$$
\begin{aligned}
\check{\vartheta}(k) & =\left[\xi^{T}(k), \xi^{T}(k-\tau(k)), \chi^{T}(k)\right]^{T}, \\
\vartheta(k)= & {\left[\check{\theta}^{T}(k), w^{T}(k), \tilde{w}^{T}(k)\right]^{T}, } \\
\mathcal{A}_{i j}= & {\left[\bar{A}_{i j}+m_{0} \tilde{A}_{i j}, \tilde{G}_{i}, \Lambda_{\ell(k)}^{m} \mathcal{C}_{i j}\right], } \\
\hat{\mathcal{A}}_{i j}= & {\left[\begin{array}{ccc}
f_{0} \bar{A}_{i j} & 0 & 0 \\
g_{0} \tilde{A}_{i j} & 0 & 0 \\
0 & f_{0} \tilde{G}_{i} & 0 \\
0 & 0 & \mathcal{G}_{L} \mathcal{C}_{j}
\end{array}\right] . }
\end{aligned}
$$

In the following, we first prove the exponential stability of the filtering error dynamic (11) with $w(k)=0$ and $\tilde{w}(k)=0$. By using Lemma 2, it follows from (19)-(22) that

$$
\begin{aligned}
& \mathbb{E}\{\Delta V(k)\} \\
\leq & \mathbb{E}\left\{\sum _ { i , j , s , t = 1 } ^ { r } h _ { i } h _ { j } h _ { s } h _ { t } \check { \vartheta } ^ { T } ( k ) \left(\mathcal{A}_{i j}^{T} P \mathcal{A}_{s t}+\hat{\mathcal{A}}_{i j}^{T} \mathcal{P} \hat{\mathcal{A}}_{s t}\right.\right. \\
& +\hat{P}) \check{\vartheta}(k)\} \\
\leq & \sum_{i, j=1}^{r} h_{i} h_{j} \check{\vartheta}^{T}(k)\left(\mathcal{A}_{i j}^{T} P \mathcal{A}_{i j}+\hat{\mathcal{A}}_{i j}^{T} \mathcal{P} \hat{\mathcal{A}}_{i j}+\hat{P}\right) \check{\vartheta}(k) \\
= & \sum_{i=1}^{r} h_{i}^{2} \check{\vartheta}^{T}(k)\left(\mathcal{A}_{i i}^{T} P \mathcal{A}_{i i}+\hat{\mathcal{A}}_{i i}^{T} \mathcal{P} \hat{\mathcal{A}}_{i i}+\hat{P}\right) \check{\vartheta}(k) \\
& +\frac{1}{2} \sum_{i, j=1, i<j}^{r} h_{i} h_{j} \check{\vartheta}^{T}(k)\left[\left(\mathcal{A}_{i j}+\mathcal{A}_{j i}\right)^{T} P\left(\mathcal{A}_{i j}+\mathcal{A}_{j i}\right)\right. \\
& \left.+\left(\hat{\mathcal{A}}_{i j}+\hat{\mathcal{A}}_{j i}\right)^{T} \mathcal{P}\left(\hat{\mathcal{A}}_{i j}+\hat{\mathcal{A}}_{j i}\right)+4 \hat{P}\right] \check{\vartheta}(k) .
\end{aligned}
$$

By applying Schur's complement Lemma, we know that $\mathbb{E}\{\Delta V(k)\}<0$ if and only if (16) and (17) are true. Furthermore, along the same way of the proof of Theorem 1 in [44], it can be concluded that the nominal filtering error dynamics (11) (with $\Delta A_{f i}(k)=0, \Delta B_{f i}(k)=0, w(k)=0$ and $\tilde{w}(k)=0)$ is exponentially mean-square stable.

Next, let us deal with the $H_{\infty}$ performance of the nominal filtering error dynamic (11) (with $\Delta A_{f i}(k)=0$ and $\left.\Delta B_{f i}(k)=0\right)$. For this purpose, we assume zero-initial condition and introduce the following index:

$$
J(n)=\mathbb{E}\left\{\sum_{k=0}^{n}\left[e^{T}(k) e(k)-\gamma^{2} v^{T}(k) v(k)\right]\right\}
$$

where $n$ is a nonnegative integer. Obviously, our aim is to show $J(n)<0$ under the zero-initial condition.

Along the trajectory of the nominal filtering error dynamics of (11) (with $\Delta A_{f i}(k)=0$ and $\Delta B_{f i}(k)=0$ ) and taking 
(23) into consideration, we have that

$$
\begin{aligned}
& J(n)=\mathbb{E} \sum_{k=0}^{n}\left[e^{T}(k) e(k)-\gamma^{2} w^{T}(k) w(k)-\gamma^{2} \tilde{w}^{T}(k) \tilde{w}(k)\right. \\
& +\Delta V(k)]-\mathbb{E} V(n+1) \\
& \leq \mathbb{E} \sum_{k=0}^{n}\left[e^{T}(k) e(k)-\gamma^{2} w^{T}(k) w(k)-\gamma^{2} \tilde{w}^{T}(k) \tilde{w}(k)\right. \\
& +\Delta V(k)] \\
& \leq \sum_{k=0}^{n} \mathbb{E}\left\{\sum _ { i , j , s , t = 1 } ^ { r } h _ { i } h _ { j } h _ { s } h _ { t } \left[\check { \vartheta } ^ { T } ( k ) \left(\mathcal{A}_{i j}^{T} P \mathcal{A}_{s t}\right.\right.\right. \\
& \left.+\hat{\mathcal{A}}_{i j}^{T} \mathcal{P} \hat{\mathcal{A}}_{s t}+\hat{P}\right) \check{\vartheta}(k)+\left(\bar{E}_{i j} \xi(k)\right)^{T} \bar{E}_{s t} \xi(k) \\
& \left.\left.-\gamma^{2} w^{T}(k) w(k)-\gamma^{2} \tilde{w}^{T}(k) \tilde{w}(k)\right]\right\} \\
& \leq \sum_{i, j, s, t}^{r} h_{i} h_{j} h_{s} h_{t} \vartheta^{T}(k)\left(\mathcal{Z}_{i j}^{T} \tilde{P} \mathcal{Z}_{s t}+\hat{\mathcal{Z}}_{i j}^{T} \overline{\mathcal{P}} \hat{\mathcal{Z}}_{s t}\right. \\
& +\bar{P}) \vartheta(k) \\
& \leq \sum_{i, j}^{r} h_{i} h_{j} \vartheta^{T}(k)\left(\mathcal{Z}_{i j}^{T} \tilde{P} \mathcal{Z}_{i j}+\hat{\mathcal{Z}}_{i j}^{T} \overline{\mathcal{P}} \hat{\mathcal{Z}}_{i j}+\bar{P}\right) \vartheta(k) \\
& =\sum_{i=1}^{r} h_{i}^{2} \vartheta^{T}(k)\left(\mathcal{Z}_{i i}^{T} \tilde{P} \mathcal{Z}_{i i}+\hat{\mathcal{Z}}_{i i}^{T} \overline{\mathcal{P}} \hat{\mathcal{Z}}_{i i}+\bar{P}\right) \vartheta(k) \\
& +\frac{1}{2} \sum_{i, j=1, i<j}^{r} h_{i} h_{j} \vartheta^{T}(k)\left[\left(\mathcal{Z}_{i j}+\mathcal{Z}_{j i}\right)^{T} \tilde{P}\left(\mathcal{Z}_{i j}+\mathcal{Z}_{j i}\right)\right. \\
& \left.+\left(\hat{\mathcal{Z}}_{i j}+\hat{\mathcal{Z}}_{j i}\right)^{T} \overline{\mathcal{P}}\left(\hat{\mathcal{Z}}_{i j}+\hat{\mathcal{Z}}_{j i}\right)+4 \bar{P}\right] \vartheta(k) .
\end{aligned}
$$

By using Schur's complement lemma, it can be concluded from (19)-(22) that $J(n)<0$. Letting $n \rightarrow \infty$, it is easy to see that

$$
\sum_{k=0}^{\infty} \mathbb{E}\left\{\|e(k)\|^{2}\right\} \leq \gamma^{2} \sum_{k=0}^{\infty}\|v(k)\|^{2},
$$

which completes the proof of Theorem 1.

Having determined the analysis results, we are now in a position to solve the fuzzy filter design problem.

Theorem 2: Given a scalar $\gamma>0$, the nominal filtering error dynamics (11) (with $\Delta A_{f i}(k)=0$ and $\Delta B_{f i}(k)=0$ ) is exponentially mean-square stable with $H_{\infty}$ the performance $\gamma$ if there exist matrices $P>0, Q>0$ and $R_{l}>0(l=$ $1,2, \cdots, L)$ and matrices $X_{j}$ satisfying

$$
\begin{aligned}
& \Gamma_{1} \triangleq {\left[\begin{array}{cc}
\bar{P} & * \\
\Sigma_{i i} & -\Phi
\end{array}\right]<0, \quad i=1,2, \cdots, r, } \\
& \Gamma_{2} \triangleq \quad {\left[\begin{array}{cc}
4 \bar{P} & * \\
\Sigma_{i j}+\Sigma_{j i} & -\Phi
\end{array}\right]<0, } \\
& i, j \in \mathbb{N}, 1 \leq i<j \leq r,
\end{aligned}
$$

where

$$
\begin{aligned}
& \Sigma_{i j}=\left[\begin{array}{cc}
\Sigma_{11 i j} & \Sigma_{12 i j} \\
\Sigma_{21 i} & \Sigma_{22 i} \\
\Sigma_{31 i j} & 0 \\
0 & \Sigma_{42 j}
\end{array}\right], \\
& \Sigma_{11 i j}=\left[\begin{array}{cc}
P \breve{A}_{0 i}+m_{0} X_{j} \breve{R}_{1} & P \tilde{G}_{i} \\
\bar{E}_{i j} & 0
\end{array}\right. \\
& \left.\begin{array}{c}
\Lambda_{\ell(k)}^{m}\left(I_{L} \otimes X_{j} \breve{R}_{2}\right) \\
0
\end{array}\right] \\
& \Sigma_{12 i j}=\left[\begin{array}{cc}
P \breve{B}_{0 i}+m_{0} X_{j} \breve{R}_{3} & \Lambda_{\ell(k)}^{m}\left(I_{L} \otimes X_{j} \breve{R}_{3}\right) \\
0 & 0
\end{array}\right], \\
& \Sigma_{21 i}=\left[\begin{array}{ccc}
f_{0} P \breve{A}_{0 i} & 0 & 0 \\
0 & 0 & 0
\end{array}\right], \Sigma_{22 i}=\left[\begin{array}{cc}
0 & 0 \\
f_{0} P \breve{B}_{0 i} & 0
\end{array}\right] \text {, } \\
& \Sigma_{31 i j}=\operatorname{diag}\left\{g_{0} X_{j} \breve{R}_{2}, f_{0} P \tilde{G}_{i}, \mathcal{G}_{L} \otimes X_{j} \breve{R}_{2}\right\} \text {, } \\
& \Sigma_{42 j}=\operatorname{diag}\left\{g_{0} X_{j} \breve{R}_{3}, \mathcal{G}_{L} \otimes X_{j} \breve{R}_{3}\right\}, \\
& \breve{A}_{0 i}=\left[\begin{array}{cc}
A_{i} & 0 \\
0 & 0
\end{array}\right], \breve{B}_{0 i}=\left[\begin{array}{c}
B_{i} \\
0
\end{array}\right] \text {, } \\
& \breve{R}_{1}=\left[\begin{array}{ll}
0 & I \\
C & 0
\end{array}\right], \breve{R}_{2}=\left[\begin{array}{ll}
0 & 0 \\
C & 0
\end{array}\right] \\
& \breve{R}_{3}=\left[\begin{array}{ll}
0 & D^{T}
\end{array}\right]^{T}, \quad \Phi=\operatorname{diag}\{P, I, \overline{\mathcal{P}}\}, \\
& \hat{I}=\left[\begin{array}{ll}
0 & I
\end{array}\right]^{T} \text {, }
\end{aligned}
$$

and $\bar{P}_{i j}$ is defined in Theorem 1. Furthermore, if $\left(P, Q_{m}, R_{l}, X_{j}\right)$ is a feasible solution of (25) and (26), then the filter parameters in the form of (10) are given as follows:

$$
\left[A_{f j}, B_{f j}\right]=\left(\hat{I}^{T} P \hat{I}\right)^{-1} \hat{I}^{T} X_{j}
$$

Proof: To avoid partitioning the positive definite matrices $P, Q_{m}$ and $R_{l}$, we rewrite the parameters in Theorem 1 in the following form:

$$
\begin{aligned}
\tilde{A}_{j} & =\hat{I} K_{j} \breve{R}_{2}, \tilde{B}_{j}=\hat{I} K_{j} \breve{R}_{3} \\
\mathcal{C}_{j} & =I_{L} \otimes \hat{I} K_{j} \breve{R}_{2}, \mathcal{D}_{j}=I_{L} \otimes \hat{I} K_{j} \breve{R}_{3}
\end{aligned}
$$

where $K_{j}=\left[A_{f j}, B_{f j}\right]$. Noticing (28), we can rewrite (19) and (21) as follows:

$$
\begin{aligned}
& {\left[\begin{array}{cc}
\bar{P} & * \\
\hat{\Sigma}_{i i} & -\Phi^{-1}
\end{array}\right]<0, \quad i=1,2, \cdots, r} \\
& {\left[\begin{array}{cc}
4 \bar{P} & * \\
\hat{\Sigma}_{i j}+\hat{\Sigma}_{j i} & -\Phi^{-1}
\end{array}\right]<0} \\
& i, j \in \mathbb{N}, 1 \leq i<j \leq r
\end{aligned}
$$


where

$$
\begin{aligned}
& \hat{\Sigma}_{i j}=\left[\begin{array}{cc}
\hat{\Sigma}_{11 i j} & \hat{\Sigma}_{12 i j} \\
\hat{\Sigma}_{21 i} & 0 \\
\hat{\Sigma}_{31 i j} & 0 \\
0 & \hat{\Sigma}_{42 j}
\end{array}\right], \\
& \hat{\Sigma}_{11 i j}=\left[\begin{array}{cc}
\breve{A}_{0 i}+m_{0} \hat{I} K_{j} \breve{R}_{1} & \tilde{G}_{i} \\
\bar{E}_{i j} & 0 \\
\Lambda_{\ell(k)}^{m}\left(I_{L} \otimes \hat{I} K_{j} \breve{R}_{2}\right) \\
0
\end{array}\right], \\
& \hat{\Sigma}_{21 i}=\left[\begin{array}{ccc}
f_{0} \breve{A}_{0 i} & 0 & 0 \\
0 & 0 & 0
\end{array}\right], \hat{\Sigma}_{22 i}=\left[\begin{array}{cc}
0 & 0 \\
f_{0} \breve{B}_{0 i} & 0
\end{array}\right] \text {, } \\
& \hat{\Sigma}_{31 i j}=\operatorname{diag}\left\{g_{0} \hat{I} K_{j} \breve{R}_{2}, f_{0} \tilde{G}_{i}, \mathcal{G}_{L} \otimes \hat{I} K_{j} \breve{R}_{2}\right\}, \\
& \hat{\Sigma}_{42 j}=\operatorname{diag}\left\{g_{0} \hat{I} K_{j} \breve{R}_{3}, \mathcal{G}_{L} \otimes \hat{I} K_{j} \breve{R}_{3}\right\} \text {. }
\end{aligned}
$$

Pre- and post-multiplying the inequalities (29) and (30) by $\operatorname{diag}\{I, \Phi\}$ and letting $X_{j}=P \hat{I} K_{j}$, we can obtain (25) and (26) readily, and the proof is then complete.

Finally, the results obtained for the nominal filtering error dynamics will be extended to non-fragile fuzzy system with ROGVs described in (11).

Theorem 3: Consider the non-fragile fuzzy filtering error dynamics (11) and let $\gamma>0$ be a given scalar. The filtering error dynamics (11) is exponentially mean-square stable with $H_{\infty}$ the performance $\gamma$ if there exist matrices $P>0, R_{l}>0$ $(l=1,2, \cdots, L), Q_{m}>0(m=1, \cdots, h)$ and $X_{j}$, and constant scalar $\varepsilon$ satisfying the following linear matrix inequalities

$$
\begin{aligned}
& {\left[\begin{array}{ccc}
\bar{\Gamma}_{1} & * & * \\
\bar{M}_{i}^{T} & -\varepsilon I & * \\
\varepsilon \bar{N} & 0 & -\varepsilon I
\end{array}\right]<0, \quad i=1,2, \cdots, r,} \\
& {\left[\begin{array}{ccccc}
\bar{\Gamma}_{2} & * & * & * & * \\
\bar{M}_{i}^{T} & -\varepsilon I & * & * & * \\
\varepsilon \bar{N} & 0 & -\varepsilon I & * & * \\
\bar{M}_{j}^{T} & 0 & 0 & -\varepsilon I & * \\
\varepsilon N & 0 & 0 & 0 & -\varepsilon I
\end{array}\right]<0,} \\
& i, j \in \mathbb{N}, 1 \leq i<j \leq r,
\end{aligned}
$$

where

$$
\begin{aligned}
& \bar{\Gamma}_{1}=\left[\begin{array}{cc}
\Gamma_{1} & 0 \\
0 & -\tilde{\mathcal{P}}
\end{array}\right], \bar{\Gamma}_{2}=\left[\begin{array}{cc}
\Gamma_{2} & 0 \\
0 & -\tilde{\mathcal{P}}
\end{array}\right], \\
& \bar{M}_{i}=\left[\begin{array}{c}
0 \\
\hat{M}_{i}
\end{array}\right], \tilde{\mathcal{P}}=I_{2 L+3} \otimes P, \\
& \hat{M}_{i}=\left[\begin{array}{llll}
\hat{M}_{1 i}^{T} & 0^{T} & \hat{M}_{2 i}^{T} & \hat{M}_{3 i}^{T}
\end{array}\right]^{T}, \bar{N}=\left[\begin{array}{ll}
\hat{N} & 0
\end{array}\right] \text {, }
\end{aligned}
$$

$$
\begin{aligned}
& \hat{N}=\operatorname{diag}\{\hat{N}_{a}, 0, \underbrace{\hat{N}_{a}, \cdots, \hat{N}_{a}}_{L}, \hat{N}_{b i}, \underbrace{\hat{N}_{b}, \cdots, \hat{N}_{b}}_{L}\}, \\
& \hat{M}_{1 i}=\left[\begin{array}{ccc}
P \hat{M}_{a i} & 0 & \bar{\beta} \Lambda_{\ell(k)}^{m}\left(I_{L} \otimes P \hat{M}_{b i}\right) \\
0 & 0 & 0
\end{array}\right.
\end{aligned}
$$

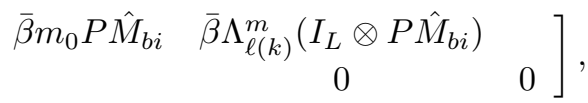

$$
\begin{aligned}
& \hat{M}_{2 i}=\operatorname{diag}\left\{\bar{\beta} g_{0} P \hat{M}_{b i}, 0, \bar{\beta} \mathcal{G}_{L} \otimes P \hat{M}_{b i},\right. \\
& \left.\bar{\beta} g_{0} P \hat{M}_{b i}, \bar{\beta} \mathcal{G}_{L} \otimes P \hat{M}_{b i}\right\} \text {, } \\
& \hat{M}_{3 i}=\operatorname{diag}\left\{\sqrt{\alpha^{*}} P \hat{M}_{c i}, 0, \sqrt{\beta^{*}} \mathcal{G}_{L}^{*} \otimes P \hat{M}_{b i},\right. \\
& \left.\sqrt{\beta^{*}} g_{0}^{*} P \hat{M}_{b i}, \sqrt{\beta^{*}} \mathcal{G}_{L}^{*} \otimes P \hat{M}_{b i}\right\}, \\
& g_{0}^{*}=\sqrt{\bar{q}_{0}+\bar{p}_{0}^{2}-m_{0}^{2}+2 \operatorname{tr}\left(\Lambda_{\ell(k)}^{d}\right)+2 d_{0} \operatorname{tr}\left(\Lambda_{\ell(k)}^{m}\right)} \text {, } \\
& g_{l}^{*}=\sqrt{2 d_{l}+2 d_{0} m_{l}+\bar{p}_{l}^{2}+2 \bar{q}_{l}-m_{l}^{2}} \text {, } \\
& \mathcal{G}_{L}^{*}=\operatorname{diag}\{\underbrace{g_{1}^{*} I, \cdots, g_{\ell(k)}^{*} I, 0, \cdots, 0}_{L}\}, \\
& \hat{M}_{a i}=\left[\begin{array}{cc}
0 & 0 \\
\bar{\beta} m_{0} M_{b i} & \bar{\alpha} M_{a i}
\end{array}\right] \text {, } \\
& \hat{M}_{b i}=\left[\begin{array}{cc}
0 & 0 \\
M_{b i} & 0
\end{array}\right], \hat{M}_{c i}=\left[\begin{array}{cc}
0 & 0 \\
0 & M_{a i}
\end{array}\right] \text {, } \\
& \hat{N}_{a}=\left[\begin{array}{cc}
N_{b} C & 0 \\
0 & N_{a}
\end{array}\right], \hat{N}_{b}=\left[\begin{array}{c}
N_{b} D \\
0
\end{array}\right],
\end{aligned}
$$

and $\Gamma_{1}$ and $\Gamma_{2}$ are defined in Theorem 2. Moreover, if $\left(P, Q_{m}, R_{l}, X_{j}, \varepsilon\right)$ is a feasible solution of (31) and (32), then the filter parameters in the form of (10) are given as follows:

$$
\left[A_{f j}, B_{f j}\right]=\left(\hat{I}^{T} P \hat{I}\right)^{-1} \hat{I}^{T} X_{j} .
$$

Proof: When $\Delta A_{f j}(k) \neq 0$ and $\Delta B_{f j}(k) \neq 0$, by using Schur's complement lemma and Lemma 2 , it follows from (19)-(22) that

$$
\begin{aligned}
& \bar{\Gamma}_{1}+\bar{M}_{i} \bar{\Delta}(k) \bar{N}+\bar{N}^{T} \bar{\Delta}^{T}(k) \bar{M}_{i}^{T}<0, \\
& \bar{\Gamma}_{2}+\bar{M}_{i} \bar{\Delta}(k) \bar{N}+\bar{N}^{T} \bar{\Delta}^{T}(k) \bar{M}_{i}^{T} \\
& +\bar{M}_{j} \bar{\Delta}(k) \bar{N}+\bar{N}^{T} \bar{\Delta}^{T}(k) \bar{M}_{j}^{T}<0
\end{aligned}
$$

where

$$
\begin{aligned}
\bar{\Delta}(k)= & \operatorname{diag}\{0, \hat{\Delta}(k)\}, \\
\hat{\Delta}(k)= & \operatorname{diag}\{\Delta(k), 0, \underbrace{\Delta(k), \cdots, \Delta(k)}_{L}, \Delta(k), \\
& \underbrace{\Delta(k), \cdots, \Delta(k)}_{L}\}, \\
\Delta(k)= & \operatorname{diag}\left\{\Delta_{2}(k), \Delta_{1}(k)\right\},
\end{aligned}
$$

and the corresponding parameters have been defined in this theorem. According to Lemma 1, we can easily obtain (31) and (32), and the details are thus omitted.

Remark 3: For the filtering error dynamics (11), a nonfragile $H_{\infty}$ filter in the form of (10) is designed in Theorem 3 such that the filtering error dynamics (11) is exponentially mean-square stable and the $H_{\infty}$ performance is satisfied. For the discrete-time stochastic system considered in this paper, there are three four main aspects which complicate the design of the $H_{\infty}$ filters, ROGVs, fading channels, time-delays and external disturbances. In Theorem 3, sufficient conditions, 
which include all of the information on four aspects, are established to ensure the expected stability and $H_{\infty}$ performance constraints. The corresponding solvability conditions for the desired non-fragile filter gains are expressed in terms of the feasibility of a set of matrix inequalities readily solved by Matlab toolbox. In the next section, an illustrative example will be provided to show the usefulness and effectiveness of the proposed design method.

\section{ILLUSTRATIVE EXAMPLE}

In this section, we present an illustrative example to demonstrate the effectiveness of the developed method. Consider a T-S fuzzy model (1) with channel fadings. The rules are given as follows:

$$
\left\{\begin{aligned}
x(k+1)= & \sum_{i=1}^{2} h_{i}\left(x_{1}(k)\right)\left[A_{i} x(k)+G_{i} x(k-\tau(k))\right. \\
& \left.+B_{i} w(k)\right] \\
y(k)= & \sum_{i=1}^{2} h_{i}\left(x_{1}(k)\right)\left[C_{i} x(k)+D_{i} w(k)\right] \\
z(k)= & \sum_{i=1}^{2} h_{i}\left(x_{1}(k)\right) E_{i} x(k)
\end{aligned}\right.
$$

The model parameters are given as follows:

$$
\begin{aligned}
& A_{1}=\left[\begin{array}{cc}
0.71440 & 0.00784 \\
-0.13616 & 0.77984
\end{array}\right], \\
& A_{2}=\left[\begin{array}{ll}
0.79416 & 0.00784 \\
0.42480 & 0.3208
\end{array}\right], \\
& G_{1}=G_{2}=\left[\begin{array}{cc}
0.0280 & 0.0040 \\
0 & 0.0188
\end{array}\right], \\
& B_{1}=B_{2}=\left[\begin{array}{ll}
0 & 0.285
\end{array}\right]^{T}, \\
& C_{1}=C_{2}=\left[\begin{array}{ll}
0.899 & 0.635
\end{array}\right], \\
& D_{1}=D_{2}=0.255, \\
& E_{1}=E_{2}=\left[\begin{array}{ll}
0.78 & 0.65
\end{array}\right], \\
& w(k)=0.4 * \operatorname{rand}(1) /(1+0.05 k) .
\end{aligned}
$$

The uncertain perturbation matrices in non-fragile fuzzy filter are given as follows:

$$
\begin{aligned}
& M_{a 1}=M_{a 2}=\left[\begin{array}{ll}
0.4 & 0.2
\end{array}\right]^{T}, \\
& M_{b 1}=M_{b 2}=\left[\begin{array}{ll}
0.46 & 0.24
\end{array}\right]^{T}, \\
& N_{a}=\left[\begin{array}{ll}
0 & 0.2
\end{array}\right], N_{b}=0.13, \\
& \Delta_{1}(k)=0.04 * \cos (k), \Delta_{2}(k)=0.04 * \sin (k) .
\end{aligned}
$$

The membership function is assumed to be

$h_{1}\left(x_{1}(k)\right)=\frac{1-\sin ^{2}\left(x_{1}(k)\right)}{2}, h_{2}\left(x_{1}(k)\right)=\frac{1+\sin ^{2}\left(x_{1}(k)\right)}{2}$, where $x_{1}(k)$ denotes the first element of the system state.

In this example, the order of the Rice fading model is $L=1$, the bounds of the channel coefficients' mathematical expectations are $\underline{p}_{0}=0.80, \bar{p}_{0}=0.90, \underline{p}_{1}=0.35$ and $\bar{p}_{1}=0.45$, the bounds of the channel coefficients' variances are $\underline{q}_{0}=0.01, \bar{q}_{0}=0.05, \underline{q}_{1}=0.06$ and $\bar{q}_{1}=0.11$. Assume that $\bar{\alpha}=0.5$ and $\bar{\beta}=0.3$.

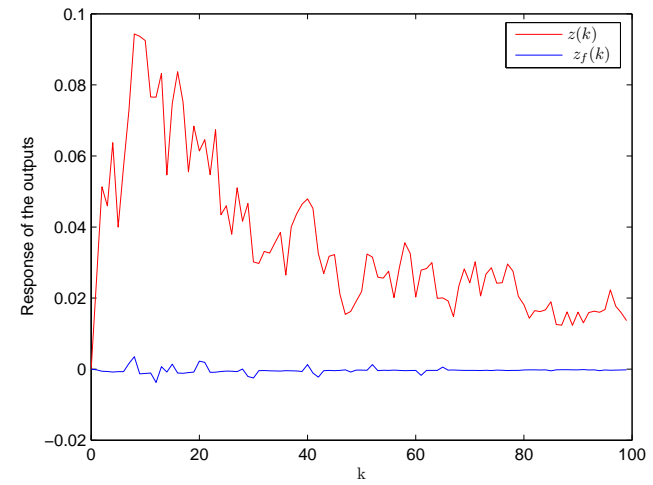

Fig. 1. Response of the outputs $z(k)$ and $z_{f}(k)$.

Our aim is to design a filter in the form of (10) such that the filtering error dynamic (11) is exponentially mean-square stable with a guaranteed $H_{\infty}$ norm bound $\gamma=1.98$. By using Theorem 3, we can obtain the following filter parameter matrices:

$$
\begin{gathered}
A_{f 1}=\left[\begin{array}{cc}
0.0064368 & 0.0000595 \\
-0.0052150 & 0.0000338
\end{array}\right], \\
A_{f 2}=\left[\begin{array}{cc}
0.0086629 & -0.0003278 \\
-0.0014637 & 0.0000187
\end{array}\right], \\
B_{f 1}=\left[\begin{array}{c}
-0.0059983 \\
0.0020822
\end{array}\right], B_{f 2}=\left[\begin{array}{c}
-0.0151666 \\
0.0022724
\end{array}\right] .
\end{gathered}
$$

Simulation results are shown in Figs. 1-3, where Fig. 1 gives the simulation results of output signal $z(t)$ and its estimation $z_{f}(t)$. Simulation results of the the disturbance input and the filtering error are shown in Fig. 2. Fig. 3 shows the response of the ratio $\sqrt{\sum_{i=0}^{k}\|e(k)\|^{2}} / \sqrt{\sum_{i=0}^{k}\|w(k)\|^{2}}$ under zero initial condition. When $y_{f}(k)=y(k)$, i.e., there is no channel fading, the simulation results of the output signal $z(t)$ and its estimation $z_{f}(t)$ are given in Fig. 4. All the simulation results have confirmed that the designed $H_{\infty}$ filter performs well.

Remark 4: In the simulation results, Fig. 3 has been added to show the response of the ratio $\sqrt{\sum_{i=0}^{k}\|e(k)\|^{2}} / \sqrt{\sum_{i=0}^{k}\|w(k)\|^{2}}$ under zero initial condition, where criterion (13) of the $H_{\infty}$ performance is satisfied. In comparison with Fig. 1, Fig. 4 shows that the non-fragile $H_{\infty}$ filter performs better without the channel fading phenomena.

\section{Conclusions}

In this paper, we have investigated the design problem of non-fragile $H_{\infty}$ filter for a class of T-S fuzzy systems with ROGVs and channel fadings. By using the Lyapunov stability theory and stochastic analysis, sufficient conditions for the exponential mean-square stability of the fuzzy filtering error dynamic have been obtained and, at the same time, the prescribed $H_{\infty}$ disturbance rejection attenuation level has been guaranteed. Then, the explicit expression of the desired filter parameters has been derived. Finally, The effectiveness of the proposed filter design is shown by a numerical simulation example. 


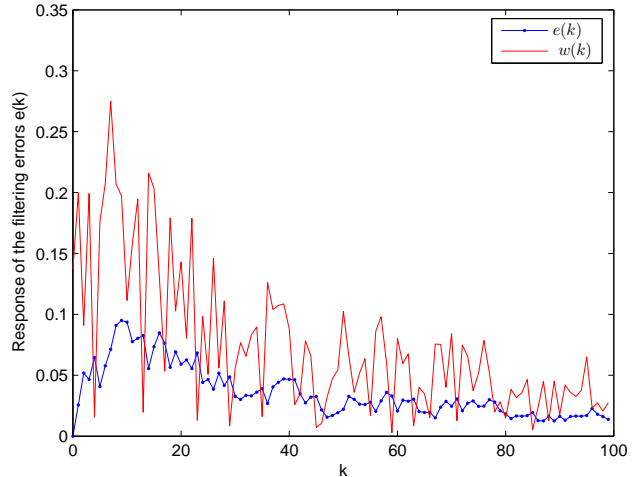

Fig. 2. Response of the filtering errors $e(k)$.

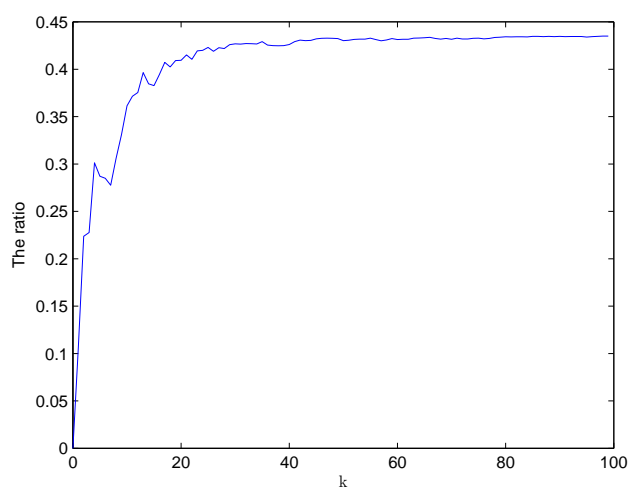

Fig. 3. Response of the ratio $\sqrt{\sum_{i=0}^{k}\|e(k)\|^{2}} / \sqrt{\sum_{i=0}^{k}\|w(k)\|^{2}}$.

\section{REFERENCES}

[1] B. D. O. Anderson and J. B. Moore, Optimal Filtering, Prentice-Hall, Englewood Cliffs, NJ, 1979.

[2] S. Boyd, L. E. Ghaoui, E. Feron, and V. Balakrishnan, Linear Matrix Inequalities in Systems and Control Theory, Philadelphia, PA: SIAM, 1994.

[3] R. G. Brown and P. Y. C. Hwang, Introduction to Random Signals and Applied Kalman Filtering, Wiley, New York, 1992.

[4] Y. Cao and P. Frank, Analysis and synthesis of nonlinear time-delay systems via fuzzy control approach, IEEE Trans. Fuzzy Syst., vol. 8, no. 2, pp. 200-211, Apr. 2000.

[5] X. Chang and G. Yang, Nonfragile $H_{\infty}$ filtering of continuous-time fuzzy systems,IEEE Trans. Signal Process., vol. 59, no. 4, pp. 15281538, Apr. 2011.

[6] X. Chang, and G. Yang, Non-fragile fuzzy $H_{\infty}$ filter design for nonlinear continuous-time systems with D stability constraints, Signal Process., vol. 92, no. 2, pp. 575-586, Feb. 2012.

[7] Y. Chu and K. Glover, Bounds of the induced norm and model reduction errors for systems with repeated scalar nonlinearities, IEEE Trans. Autom. Control, vol. 44, no. 3, pp. 471-483, Mar. 1999.

[8] Y. Chu, Further results for systems with repeated scalar nonlinearities,IEEE Trans. Autom. Control, vol. 44, no. 12, pp. 2031-2035, Dec. 2001.

[9] B. Chen and X. Liu, Delay-dependent robust $H_{\infty}$ control for T-S fuzzy systemswith time delay, IEEE Trans. Fuzzy Syst., vol. 13, no. 4, pp. 544556, Aug. 2005.

[10] M. Chen, G. Feng, H. Ma, and G. Chen, Delay-dependent $H_{\infty}$ filter design for discrete-time fuzzy systems with time-varying delays, IEEE Trans. Fuzzy Syst., vol. 17, no. 3, pp. 604-616, Jun. 2009.

[11] S. F. Derakhshan and A. Fatehi, Non-Monotonic Lyapunov Functions for Stability Analysis and Stabilization of Discrete Time Takagi-Sugeno Fuzzy Systems, Int. J. Innov. Comput. Inf. Control, vol. 10, no. 4, pp. 1567-1586, Aug. 2014.

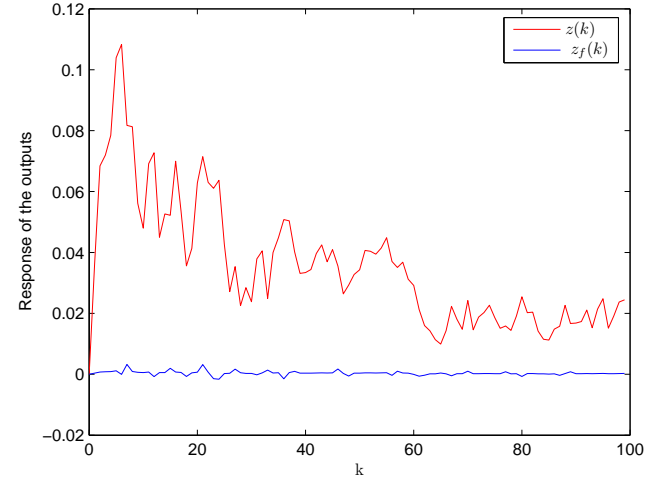

Fig. 4. Response of the outputs $z(k)$ and $z_{f}(k)$ without channel fadings.

[12] D. Ding, X. Li, Z. Shi, and X. Guo, Nonfragile $H_{\infty}$ filtering for discretetime T-S fuzzy systems, Prcoceedings of the 31th Chinese Control Conference, Hefei, China, pp. 3552-3557, Jul. 2012.

[13] H. Dong, Z. Wang, D. W. C. Ho, and H. Gao, Robust $H_{\infty}$ fuzzy outputfeedback control with multiple probabilistic delays and multiple missing measurements, IEEE Trans. Fuzzy Syst., vol. 18, no. 4, pp. 712-725, Aug. 2010.

[14] H. Dong, Z. Wang, J. Lam, and H. Gao, Fuzzy-model-based robust fault detection with stochastic mixed time delays and successive packet dropouts, IEEE Trans. Syst., Man, Cybern. B, Cybern., vol. 42 , no. 2, pp. 365-376, Apr. 2012.

[15] N. Elia, Remote stabilization over fading channels, Syst. Control Lett., vol. 54, no. 3, pp. 237-249, Mar. 2005.

[16] E. Garone, B. Sinopoli, A. Goldsmith, and A. Casavola, LQG control for MIMO systems over multiple erasure channels with perfect acknowledgment, IEEE Trans. Autom. Control, vol. 57, no. 2, pp. 450-456, Feb. 2012.

[17] M. Fang and J. H. Park, Non-fragile synchronization of neural networks with time-varying delay and randomly occurring controller gain fluctuation, Applied Mathematics and Computation,vol. 219, no. 15, pp. 80098017, Apr. 2013.

[18] H. Gao, Y. Zhao, and T. Chen, $H_{\infty}$ fuzzy control with missing data, in Proc. IEEE Int. Conf. Syst., Man, Cybern., Montreal, Que., Canada, pp. 801-806, Oct. 2007.

[19] H. Gao, Y. Zhao, J. Lam, and K. Chen, $H_{\infty}$ fuzzy filtering of nonlinear systems with intermittent measurements, IEEE Trans. Fuzzy Syst., vol. 17, no. 2, pp. 291-300, Apr. 2009.

[20] X. Guan and C. Chen, Delay-dependent guaranteed cost control for T-S fuzzy systems with time delays, IEEE Trans. Fuzzy Syst., vol. 12, no. 2, pp. 236-249, Apr. 2004.

[21] S. He and F. Liu, Finite-Time $H_{\infty}$ fuzzy control of nonlinear jump systems with time delays via dynamic observer-based state feedback, IEEE Trans. Fuzzy Syst., vol. 20, no. 4, pp. 605-614, Aug. 2012.

[22] J. Hu, Z. Wang, H. Gao, and L.K. Stergioulas, Robust sliding mode control for discrete stochastic systems with mixed time delays, randomly occurring uncertainties, and randomly occurring nonlinearities, IEEE Trans. Ind. Electron., vol. 59, no. 7, pp. 3008-3016, Jul. 2012.

[23] C. Huang, D.W.C. Ho, and J. Lu, Partial-Information-Based Distributed Filtering in Two-Targets Tracking Sensor Networks IEEE Transactions on Circuits and Systems I, vol. 59 , no, 4, pp.820-813, Apr. 2012.

[24] L. H. Keel and S. P. Bhattacharyya, Robust, fragile, or optimal? IEEE Trans. Automatic Contr., vol. 42, no. 8, pp. 1098-1105, Jun. 1997.

[25] W. J. Kickert and E. H. Mamdani, Analysis of a fuzzy logic controller, Fuzzy Sets Syst., vol. 1, no. 1, pp. 29-44, Jan. 1978.

[26] B. Jiang, Z. Mao, and P. Shi, $H_{\infty}$ filter design for a class of networked control systems via T-S fuzzy-model approach, IEEE Trans. Fuzzy Syst., vol. 18, no. 1, pp. 201-208, Aug. 2010.

[27] H. Z. Li and M. Y. Fu, A linear matrix inequality approach to robust $H_{\infty}$ filtering, IEEE Trans. Signal Process., vol. 45, no. 9, pp. 23382350, Sep. 1997.

[28] F. Li, P. Shi, L. Wu, and X. Zhang, Fuzzy-model-basedd Dstability and nonfragile control for discrete-time descriptor systems with multiple delays, IEEE Trans. Fuzzy Syst., vol. 22, no. 4, pp. 1019-1025, Aug. 2014. 
[29] M. S. Mahmoud, Resilient linear filtering of uncertain systems, Automatica, vol. 40, no. 10, pp. 1797-1802, Oct. 2004

[30] X. Mao, Stochastic Differential Equations and Applications, Chichester, Horwood Publishing, 1997.

[31] Y. Mostofi and R. M. Murray, To drop or not to drop: design principles for Kalman filtering over wireless fading channels, IEEE Trans. Autom. Control, vol. 54, no. 2, pp. 376-381, Feb. 2009.

[32] S. K. Nguang and W. Assawinchaichote, $H_{\infty}$ filtering for fuzzy dynamical systems with D-stability constraints, IEEE Trans. Circuits Syst. I, vol. 50, no. 11, pp. 1503-1508, Nov. 2003.

[33] J. Qiu, G. Feng, and J. Yang, A new design of delay-dependent robust $H_{\infty}$ filtering for discrete-time T-S fuzzy systems with time-varying delay, IEEE Trans. Fuzzy Syst., vol. 17, no. 5, pp. 1044-1058, Oct. 2009.

[34] D. E. Quevedo, A. Ahlén, A. S. Leong, and S. Dey, On Kalman filtering over fading wireless channels with controlled transmission powers, Automatica, vol. 48, no. 7, pp. 1306-1316, Jul. 2012.

[35] B. Shen, Z. Wang, H. Shu, and G. Wei, $H_{\infty}$ filtering for nonlinear discrete-time stochastic systems with randomly varying sensor delays, Automatica, vol. 45, no. 4, pp. 1032-1037, Apr. 2009.

[36] Q. Shen, B. Jiang and V. Cocquempot, Fault-tolerant control for T-S fuzzy systems with application to near-space hypersonic vehicle with actuator faults, IEEE Trans. Fuzzy Syst., vol. 20, no. 4, pp. 652-665, Aug. 2012.

[37] P. Shi, E. K. Boukas, and R. Agarwal, Kalman filtering for continuous time uncertain systems with Markovian jumping parameters, IEEE Trans. Autom. Control, vol. 44, no. 8, pp. 1592-1597, Aug. 1999.

[38] P. Shi, X. Luan and F. Liu, $H_{\infty}$ filtering for discrete-time systems with stochastic incomplete measurement and mixed delays, IEEE Trans on Industrial Electronics, vol. 59, no. 6, pp. 2732-2739, Jun. 2012.

[39] M. Sugeno, Industrial applications of fuzzy control, New York, Elsevier, 1985.

[40] X. Su, P. Shi, L. Wu, and Y.D. Song, A novel approach to filter design for T-S fuzzy discrete-time systems with time-varying delay, IEEE Trans. Fuzzy Syst., vol. 20, no. 6, pp. 1114-1129, Dec. 2012.

[41] T. Takagi and M. Sugeno, Fuzzy identification of systems and its application to modelling and control, IEEE Trans. Syst., Man, Cybern., vol. SMC-15, no. 1, pp. 116-132, Jan./Feb. 1985.

[42] K. Tanaka and M. Sugeno, Stability analysis and design of fuzzy control systems, Fuzzy Sets Syst., vol. 45, no. 2, pp. 135-156, Jan. 1992.

[43] K. Tanaka and H. O. Wang, Fuzzy Control Systems Design and Analysis: A LMI Approach. New York: Wiley, 2001.

[44] Z. Wang, D. W. C. Ho, Y. Liu, and X. Liu, Robust $H_{\infty}$ control for a class of nonlinear discrete time-delay stochastic systems with missing measurements, Automatica, vol. 45, no. 3, pp. 684-691, Mar. 2009.

[45] L. Wu and Z. Wang, Fuzzy filtering of nonlinear fuzzy stochastic systems with time-varying delay, Signal Processing, vol. 89, no. 9, pp. 17391753, Sep. 2009.

[46] L. Wu, X. Su, P. Shi, and J. Qiu, Model approximation for discrete-time state-delay systems in the T-S fuzzy framework, IEEE Trans. Fuzzy Syst., vol. 19, no. 2, pp. 366-378, Apr. 2011.

[47] N. Xiao, L. Xie, and L. Qiu, Feedback stabilization of discrete-time networked systems over fading channels, IEEE Trans. Autom. Control, vol. 57, no. 9, pp. 2176-2189, Sep. 2012.

[48] L. Xie, C. de Souza, and M. Fu, $H_{\infty}$ estimation for discrete time linear uncertain systems, Int. J. Robust and Nonlinear Control, vol. 1, no. 2, pp. 111-123, Apr./Jun. 1991.

[49] G. Yang, and W. Che, Non-fragile $H_{\infty}$ filter design for linear continuous-time systems, Automatica, vol. 44, no. 11, pp. 2849-2856, Nov. 2008.

[50] H. Zhang, H. Zhong, and C. Dang, Delay-dependent decentralized $H_{\infty}$ filtering for discrete-time nonlinear interconnected systems with timevarying delay based on the T-S fuzzy model, IEEE Trans. Fuzzy Syst., vol. 20, no. 3, pp. 431-443, Jun. 2012.

[51] J. Zhang and Y. Xia, New LMI approach to fuzzy $H_{\infty}$ filter designs, IEEE Trans. Circuits Syst. II, Exp. Briefs, vol. 56, no. 9, pp. 739-743, Sep. 2009.

[52] S. Zhou, G. Feng, J. Lam, S. Xu, Robust $H_{\infty}$ control for discretetime fuzzy systems via basis-dependent Lyapunov functions, Information Sciences, vol.174, no. 3-4, pp. 197-217, August. 2005.

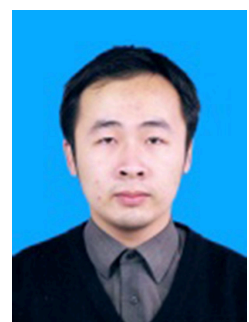

Sunjie Zhang received the B.Sc. degree in applied mathematics from Donghua University, Shanghai, China, in 2010 .

$\mathrm{He}$ is now pursuing the Ph.D. degree in control science and engineering with the Donghua University, Shanghai. His research interests include nonlinear stochastic control and filtering, as well as wireless sensor networks. He is a reviewer for some international journals.

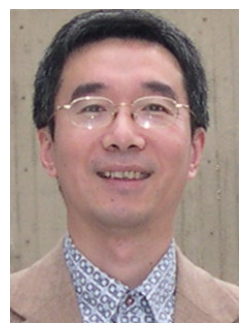

Zidong Wang was born in Jiangsu, China, in 1966. He received the B.Sc. degree in mathematics in 1986 from Suzhou University, Suzhou, China, and the M.Sc. degree in applied mathematics in 1990 and the $\mathrm{Ph}$.D. degree in electrical engineering in 1994, both from Nanjing University of Science and Technology, Nanjing, China

$\mathrm{He}$ is currently Professor of Dynamical Systems and Computing in the Department of Computer Science, Brunel University London, U.K. From 1990 to 2002, he held teaching and research appointments in universities in China, Germany and the UK. Prof. Wang's research interests include dynamical systems, signal processing, bioinformatics, control theory and applications. He has published more than 200 papers in refereed international journals. He is a holder of the Alexander von Humboldt Research Fellowship of Germany, the JSPS Research Fellowship of Japan, William Mong Visiting Research Fellowship of Hong Kong.

Prof. Wang is a Fellow of the IEEE. He is serving or has served as an Associate Editor for 12 international journals, including IEEE Transactions on Automatic Control, IEEE Transactions on Control Systems Technology, IEEE Transactions on Neural Networks, IEEE Transactions on Signal Processing, and IEEE Transactions on Systems, Man, and Cybernetics - Systems. He is also a Fellow of the Royal Statistical Society and a member of program committee for many international conferences.

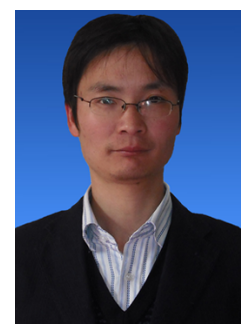

Derui Ding received the B.Sc. degree in industry engineering and the M.Sc. degree in detection technology and automation equipments from Anhui Polytechnic University, Wuhu, China, in 2004 and 2007, and the Ph.D. degree in control theory and control engineering from Donghua University, Shanghai, China, in 2014.

From July 2007 to December 2014, he was a teaching assistant and then a lecturer in the Department of Mathematics, Anhui Polytechnic University, Wuhu, China. He is currently an associate professor with the Department of Control Science and Engineering, University of Shanghai for Science and Technology, Shanghai, China. From June 2012 to September 2012, he was a Research Assistant in the Department of Mechanical Engineering, the University of Hong Kong, Hong Kong. From March 2013 to March 2014, he was a Visiting Scholar in the Department of Information Systems and Computing, Brunel University London, U.K. His research interests include nonlinear stochastic control and filtering, as well as multi-agent systems and sensor networks. He has published around 15 papers in refereed international journals. He is a very active reviewer for many international journals. 


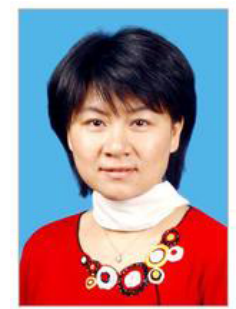

Hongli Dong received the Ph.D. degree in control science and engineering from Harbin Institute of Technology, Harbin, China, in 2012.

From July 2009 to January 2010, she was a Research Assistant with the Department of Applied Mathematics, City University of Hong Kong, Kowloon, Hong Kong. From October 2010 to January 2011, she was a Research Assistant with the Department of Mechanical Engineering, The University of Hong Kong, Pokfulam, Hong Kong. From January 2011 to January 2012, she was a Visiting Scholar with the Department of Information Systems and Computing, Brunel University, Uxbridge, U.K. She is currently with the College of Electrical and Information Engineering, Northeast Petroleum University, Daqing, China. She is an active Reviewer for many international journals. Her current research interests include robust control and networked control systems.

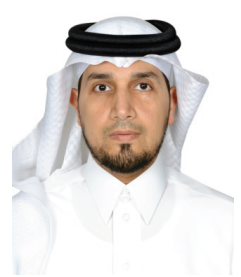

Fuad E. Alsaadi received the B.S. and M.Sc. degrees in electronic and communication from King AbdulAziz University, Jeddah, Saudi Arabia, in 1996 and 2002. He then received the Ph.D. degree in Optical Wireless Communication Systems from the University of Leeds, Leeds, UK, in 2011. Between 1996 and 2005, he worked in Jeddah as a communication instructor in the College of Electronics \& Communication. He is currently an assistant professor of the Electrical and Computer Engineering Department within the Faculty of Engineering, King Abdulaziz University, Jeddah, Saudi Arabia. He published widely in the top IEEE communications conferences and journals and has received the Carter award, University of Leeds for the best $\mathrm{PhD}$. He has research interests in optical systems and networks, signal processing, synchronization and systems design.

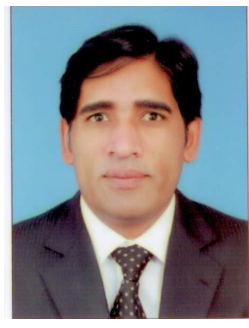

Tasawar Hayat did his Ph.D. in applied mathematics from Quaid-i-Azam University, Islamabad, Pakistan in 1999. Since then, he has been in teaching and research at Quaid-i-Azam University, Islamabad, Pakistan. He joined as lecturer in 1998, and has been promoted to Professor. Besides that, he has been appointed as Distinguished National Professor by the Higher Commission of Pakistan. He has published extensively in Newtonian and non-Newtonian fluid mechanics, mathematical modelling, signal processing and wave motion. He has received many national and international awards, and is well known internationally. He is a Highly Cited Researcher. 